\title{
TITLE:
}

\section{A Far-Infrared H-R Diagram of Young Stellar Objects}

$\operatorname{AUTHOR}(\mathrm{S})$ :

Djamaluddin, Thomas; Saito, Mamoru

\section{CITATION:}

Djamaluddin, Thomas ...[et al]. A Far-Infrared H-R Diagram of Young Stellar Objects. Memoirs of the Faculty of Science, Kyoto University. Series of physics, astrophysics, geophysics and chemistry 1995, 39(2): 197-237

\section{ISSUE DATE:}

1995-03

URL:

http://hdl.handle.net/2433/257632

RIGHT: 
Memoirs of the Faculty of Science, Kyoto University, Series of Physics, Astrophysics, Geophysics and Chemistry, Vol. XXXIX, No. 2, Article 2, 1995

\title{
A Far-Infrared H-R Diagram of Young Stellar Objects
}

\author{
By \\ Thomas DJAMALUDDIN and Mamoru SATTÖ \\ Department of Astronony, Faculty of Science, Kyoto University \\ Kyoto 606-01, Japan \\ (Receized Nowember 25, 199:)
}

\begin{abstract}
The observed spectral energy distributions of high-and low-mass young stellar objects (YSOs) are almost a modified blackbody-like one at far-infrared (FIR) to millimeter wavelengths. These emissions are produced at the outer envelopes of YSOs and the spectra are represented by the FIR color made by the IRAS flux densities at 60 and $100 \mu \mathrm{m}, f_{60}$ and $f_{100}$. For the nearby star-forming regions in Per-OB2, Taurus, Orion, Vela, and $\rho$ Ophiucus molecular clouds, we selected cold IRAS point sources corresponding to YSOs by using the color criterion of $f_{60}>f_{12}$, and constructed FIR H-R diagrams of YSOs, i.c., the luminosity of $60 \mu \mathrm{m}$ versus the color $\log \left(f_{100} / f_{60}\right)$ relation. We also examined the visual counterparts of these YSOS on the POSS prints. The FIR H-R diagrams for the individual star-forming regions show common properties in the luminosity-color relation and the locations of the extreme Class I YSOs and the active YSOs. The fundamental line on the FIR H-R diagram is a constant emelope mass sequence (CEMS) along which YSOs move following the evolutionary change in the luminosity of the central object. From analysis of the FIR H-R diagrams, we suggest that the extreme Class I YSOs have already stored most of material of stellar mass in the central part and are forming stars through a slow accretion of the material.
\end{abstract}

\section{Introduction}

In a star forming molecular cloud, there are many young stellar objects (hereafter YSOs) with various masses in various evolutionary stages (e.g., Myers et al. 1987; Strom, Strom \& Merrill 1993). Our knowledge about evolutionary process of YSOs is still incomplete in the sense that we do not know observational parameters specifying both the evolutionary stage of such YSOs and the resultant stellar mass which is the fundamental parameter of stars.

Hydrodynamical studies claim that molecular cores collaspe with increasing of luminosity, and the mass concentrated into the central part forms protostar and the surrounding disk (e.g., Larson 1977; Terebey, Shu \& Cassen 1984; Shu, Adams \& Lizano 1987). YSOs radiate mainly infrared emission with broad spectral energy distributions (SEDs) with the variety in extension shortward of mid-infrared wavelength; the emissions at mid-and near-infrared wavelengths come from the inner objects consisting of star and the surrounding disk and the SEDs spread towards shorter wavelengths with time, as the inner objects evolve and the outer envelope disperses (e.g., Wynn-Williams 1982; Chini, Krügel \& Kreysa 1986; Adams, Lada \& Shu 1987; 
Lada 1987; Myers et al. 1987 ; Shu et al. 1987; Kenyon, Calvet \& Hartmann 1993). The SEDs are thus considered to indicate evolutionary stages of YSOs; the indicators proposed so far are the slope between mid- and near-infrared wavelength (Lada 1987) and the mean frequency (Ladd et al. 1991).

It is recently recognized that the observed YSOs spectra from FIR to millimeter, i.e., the longer wavelength part of the broad SEDs, fit a modified blackbody radiation with a peak around $100 \mu \mathrm{m}$ for both high- and low-mass YSOs (e.g., Beckwith et al. 1986; Chini et al. 1986; Davidson 1987; Wilking et al. 1989; Walker, Adams \& Lada 1990, hereafter WAL; Ladd et al. 1991); that is, the flux densities are proportional to $\nu^{\beta} B_{\nu}(T)$, where $B_{\nu}(T)$ is the Planck function for a temperature $T-20$ to $50 \mathrm{~K}$ and $\nu^{\beta}$ is proportional to a dust emissivity with $\beta \sim 1$ to 2 . WAL interpret the observed spectra of 12 star-forming molecular cores by a model of isothermal dusty envelopes, by adopting probable values of three parameters, $T(\simeq 25$ to $61 \mathrm{~K}), \beta(\simeq 0.87$ to 1.79$)$, and apparent size. Figure 1 shows that the WAL's temperatures of 11 objects, for which WAL performed the fitting for the spectra measured at 56 to $3,350 \mu \mathrm{m}$, are correlated with the color $[100-60] \equiv \log \left(f_{100} / f_{60}\right)$ for the $I R A S$ flux densities at $100 \mu \mathrm{m}$ and $60 \mu \mathrm{m}$ and the color temperatures for $\beta=1$. Figure 1 suggests that the IRAS color [100-60] represents some average temperature of the outer envelope of YSOs (Ellis et al. 1990).

When a dust grain in optically thin envelope of YSOs is illuminated by radiation from the central objects, its temperature, $T_{d}$, depends mainly on the luminosity, $L *$, of the central objects and radius, $r$, of envelope as

$$
T_{d}=\left[L_{*} T_{*}^{\beta} /\left(16 \pi \sigma r^{2}\right)\right]^{1 /(4+\beta)},
$$

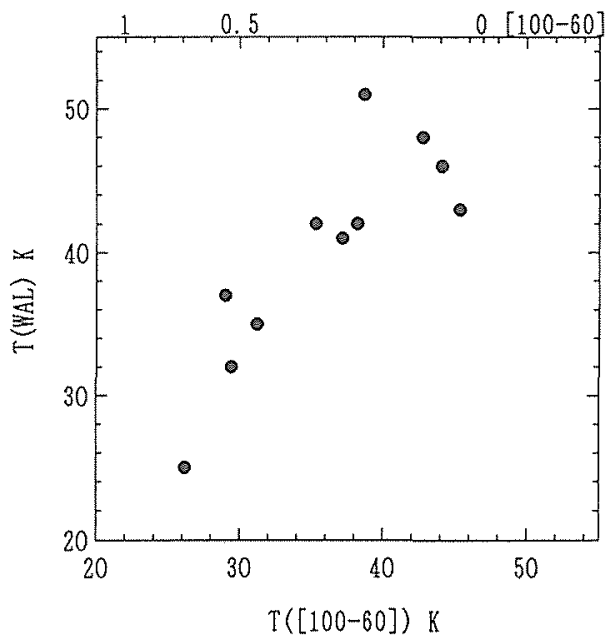

Fig. 1. Color temperatures of YSOs, $T_{100 / 60}$, derived from IRAS flux densities at $60 \mu \mathrm{m}$ and $100 \mu \mathrm{m}$ versus dust temperatures, $T_{W A L}$, derived by Walker, Adams \& Lada (1990). The flux density of dust cmission is assumed to be proportional to $\nu^{\beta} B_{\nu}(T)$, where $B_{\nu}$ is the Planck function and $\beta$ is a constant. The color temperatures are derived for $\beta=1$, while $T_{W_{A} L}$ were derived for fitting of the spectra measured at wavelengths of 58 to 3,350 $\mu \mathrm{m}$, in which the values of $\beta$ and dust temperature are free parameters. 
where $\beta$ is a constant in dust emissivity of $\propto \nu^{\beta}$ and the value is 1 to 2 (Hildebrand 1983), $T_{*}$ is the effective temperature of the central object, and $\sigma$ is the StefanBoltzmann constant (Scoville \& Kwan 1976; Beckwith et al. 1986). During the collapsing phase, $L *$ remarkably increases with time, while the radius of outer envelope is nearly constant (e.g., Shu 1977; Terebey et al. 1984). Therefore, evolution of a YSO in the collapsing phase may be traced by a locus on $T_{d}-L_{*}$ plane, along which $T_{d}$ and $L_{*}$ both become higher with time. The values of $L *$ and $r$ both are larger for highermass YSOs and the loci of YSOs on the $T_{d}-L_{*}$ plane separate with YSOs' mass.

Numerical caluculations of radiative transfer in spherical, dusty protostellar envelopes were carried out by Scoville \& Kwan (1976), Rowan-Robinson (1980), Yorke (1980), Yorke \& Shustov (1981), Adams \& Shu (1985), Gürtler et al. (1991), Kenyon et al. (1993), and others. The main parameters are stellar (or central object) mass and luminosity, dust properties, and mass and density distribution of envelope. The resulting spectra in FIR to millimeter wavelengths are similar for probable values of these parameters and always show a peak at FIR. The slopes of SEDs at $\sim 100 \mu \mathrm{m}$ to $1 \mathrm{~mm}$ correspond to those for $\beta \sim 1$ (Yorke \& Shustov 1981), and the color temperatures do not change at 50 to $100 \mu \mathrm{m}$ with wavelength and thus the spectra look like a blackbody in spite of optically thin envelopes at these wavelengths (Rowan-Robinson 1980). These features are consistent with the above-mentioned observed features. As the envelope mass is less, the FIR peak moves toward shorter wavelength (Yorke 1980; Kenyon et al. 1993). If the envelope mass is fixed and the luminosity increases, the FIR peak moves also toward shorter wavelength (Kenyon et al. 1993). The effective radius at FIR does not change with time during the evolutionary phase with optical depth of 1,000 to 10 at $0.1 \mu \mathrm{m}$ (Yorke \& Shustov 1981). Based on the radiative transfer calculations, Chini et al. (1986), Churchwell, Wolfire \& Wood (1990), and Kenyon et al. (1993) found the structures of the envelope and central disk reproducing the observed SEDs.

These observed and calculated spectral features of YSOs at FIR suggest that a FIR H-R diagram of YSOs may describe early phase of evolution of YSOs, although any H$\mathrm{R}$ diagram has been considered to be impossible (Beichman et al. 1986; Adams 1990; Lada 1991; Myers \& Ladd 1993). In this paper we propose a FIR H-R diagram of YSOs for each star-forming region by using IRAS flux densities at $60 \mu \mathrm{m}$ and $100 \mu \mathrm{m}$; the color is $[100-60] \equiv \log \left(f_{100} / f_{60}\right)$ and the luminosity is $L_{60} \equiv d^{2} f_{60}$, where $d$ is the distance to the star forming region. The reasons that we use these parameters are as follows :

(1) The emissions at $60 \mu \mathrm{m}$ and $100 \mu \mathrm{m}$ originate at almost the same region in outer envelope of a YSO; the envelope extends outside a near-infrared photosphere surrounding the central objects (e.g., Stahler, Shu \& Taam 1980) and the color [100-60] represents some average dust temperature at the envelope (e.g., Chini et al. 1986; Butner et al. 1990; Ellis et al. 1990). We call the region the FIR emitting envelope. Note that the color temperature corresponding to [100-60] follows a change of a total luminosity of YSO (see equation (1)), rather than the FIR luminosity which depends on dust mass in the envelope as well as its dust temperature. 
(2) The FIR emitting envelope is optically thin at $60 \mu \mathrm{m}$ and $100 \mu \mathrm{m}$ (e.g. Harvey et al. 1979; Kenyon et al. 1993; Natta et al. 1993), and thus its fluxes are independent on the geometry of YSOs, although the observed features in near-infrared wavelength depend on the geometry.

(3) The flux densities at $60 \mu \mathrm{m}$ is more free from diffuse component than those at $100 \mu \mathrm{m}$, and sensitively change with dust temperature because $60 \mu \mathrm{m}$ tends to be at the side of the Wien distribution of the modified blackbody-like SEDs.

(4) IRAS provides us unbiased survey data of YSOs in almost all nearby starforming regions.

In $\$ 2$ we construct FIR H-R diagrams of YSOs for nearby star-forming regions and describe properties of the FIR H-R diagrams. In $\$ 3$ we discuss mass of the FIR emitting envelope and an evolutionary track of YSOs on the FIR H-R diagram. A summary is given $\$ 4$.

\section{Far-Infrared H-R Diagrams of $\mathrm{YSO}$}

\subsection{Selection of Star-Forming Regions and Cold IRAS Point Sources}

YSOs are detected as cold IRAS point sources in star-forming regions (Beichman et al. 1986; Wood \& Churchwell 1989). In a star-forming region, YSOs form in some mass range and time span under similar environment. We thus consider that a FIR H$R$ diagram for each star-forming region contains essential information of evolution of YSOs. We here treat six well-known, nearby star forming regions in Perseus-OB2, Taurus, Orion A and B, Vela, and $\rho$-Ophiuchus molecular clouds; these are within 700 pc in distance and each contains 50 or more cold IRAS point sources.

We define cold IRAS point sources in IRAS Point Source Catalog (IRAS PSC) using the flux densities at 12 and $60 \mu \mathrm{m}$; the color criterion is $f_{12}<f_{60}$ and the source is so bright that $f_{60}$ has good or moderate flux quality $\left(\mathrm{FQ}_{60} \geq 2\right)$. The color criterion selects all kinds of YSOs such as T Tauri stars with residual envelope, massive YSOs with compact H II region, and other YSOs embedded in molecular cloud cores (e.g., Beichman et al. 1986 ; Emerson 1987; Harris, Clegg \& Hughes 1988; Wilking, Lada \& Young 1989; Wood \& Churchwell 1989). We chose the cold IRAS point sources still located on the parent $\mathrm{CO}$ clouds. We also made a visual search for optical counterparts of the selected sources on the POSS prints; the procedure is similar with Yamada et al. (1993) in searching for IRAS galaxies. If a source is associated with a galaxy or a planetary nebula in our visual inspection or if the association with non-YSOs, including main-sequence star, is denoted in literature, we omit it from our sample.

The upper limit, 2', in size of IRAS point sources at $100 \mu \mathrm{m}$ (IRAS Explanatory Supplement 1988) corresponds to $0.1 \mathrm{pc}$ at the nearest star forming regions in Taurus and $\rho$-Ophiuchus where low-mass stars are forming. The sizes of extended components of YSOS and T-Tauri stars in these regions have been measured using molecular lines and millimeter continuum, and they are smaller than $0.02 \mathrm{pc}$ (e.g., André et al. 1990; Ohashi et al. 1991). The radius of the FIR emitting region derived from equation ( 1$)$ is $4.3 \times 10^{-4}\left(T_{*} / T_{d}\right)^{\beta / 2}\left(30 / T_{d}\right)^{2}\left(L_{I R} / L_{\odot}\right)^{1 / 2} \mathrm{pc}$; for $T_{d}=20-50 \mathrm{~K}$, $T_{*}=300-600 \mathrm{~K}$, and $\beta=1-2$, the radius is almost less than 0.1 pc for $L_{I R}<20 L_{\odot}$. 
Thus isolated YSOs brighter than the sensitivity limit of IRAS observations are detected as IRAS point sources, and some confused sources may be identified as extended sources. At the Orion molecular clouds whose distance is about $500 \mathrm{pc}$, the critical size of IRAS point sources is $0.3 \mathrm{pc}$ and it is comparable to the typical diameters of CSS $(J=2-1)$ molecular cores (Lada, Bally \& Stark 1991 ; Tatematsu et al. 1993). The radius of the FIR emitting region is less than $0.3 \mathrm{pc}$ for $L_{I R}<200 L_{\odot}$. Most of YSOs in Orion are thus identified as IRAS point sources. The brighest infrared sources OMCI and OMG2 in Orion A are, respectively, an IRAS extended source and an IRAS point source with an upper limit flux density at $60 \mu \mathrm{m}$ and are not contained in our objects. These are complexes of two or more YSOs (Mezger, Wink \& Zylka 1990). The Vela star forming region is at $700 \mathrm{pc}$ (Liseau et al. 1992).

The most luminous infrared sources in each star forming region often correspond to clustering of YSOs where the projected surface densities of YSOs are up to $-100 \mathrm{pc}^{-2}$ (e.g., Lada et al. 1991 b; Strom et al. 1993; Carpenter et al. 1993). Some of these objects are identified as IRAS extended sources, such as OMCl. The beam size of IRAS observations at $100 \mu \mathrm{m}$ is $0.3 \mathrm{pc}$ at the Orion and $0.4 \mathrm{pc}$ at the Vela, and it is still possible that some brightest IRAS point sources selected are the complexes of several or more YSOs. We examine the complexness of such IRAS point sources by using IRAS $P S C$ and literature.

The selected star-forming regions are summarized in Table 1 as well as the adopted extents in Galactic coordinate and the numbers of the selected IRAS point sources. The sky distributions of the selected sources are shown in Figure 2. In order to avoid contamination by objects in adjacent star-forming regions, we restricted the range of

Table 1. The Selected Star-Forming Regions and Number of Cold IRAS Point Sources

\begin{tabular}{|c|c|c|c|c|c|c|}
\hline \multirow{2}{*}{ Name } & \multicolumn{2}{|c|}{ Galatic coordinate } & \multirow{2}{*}{$\begin{array}{l}\text { Area } \\
\left(\text { deg }^{2}\right)\end{array}$} & \multirow{2}{*}{$\begin{array}{c}d^{1)} \\
(\mathrm{kpc})\end{array}$} & \multirow{2}{*}{ Number $^{2}$} & \multirow{2}{*}{$\begin{array}{l}\text { Density } \\
\left(\text { deg }^{-2}\right)\end{array}$} \\
\hline & $l_{\min }, l_{\max }$ & $b_{\min }, b_{\max }$ & & & & \\
\hline PER-OB2 & 156,162 & $-24,-16$ & 9.6 & 0.35 & $(8: 27: 8: 41)$ & 8.8 \\
\hline TAURUS & 169,177 & $-19,-12$ & 36.2 & 0.14 & $\begin{array}{c}54 \\
(19: 19: 12: 4)\end{array}$ & 1.5 \\
\hline ORION B & 204,209 & $-17.5,-12.5$ & 13.5 & 0.5 & $\begin{array}{c}89 \\
(15: 19: 19: 36)\end{array}$ & 6.7 \\
\hline ORION A & 205,215 & $-21,-17.5$ & 24.3 & 0.5 & $\begin{array}{c}147 \\
(30: 29: 40: 48)\end{array}$ & 6.0 \\
\hline VELA & 260,266 & $-0.5,3$ & 16.3 & 0.7 & $\begin{array}{c}179 \\
(42: 38: 38: 61)\end{array}$ & 11.0 \\
\hline$\rho-\mathrm{Oph}$ & 351,359 & 12,20 & 17 & 0.125 & $\begin{array}{c}84 \\
(4: 25: 12: 43)\end{array}$ & 5.0 \\
\hline
\end{tabular}

1) The distances are adopted following Ungerechts and Thaddeus (1987) for Per-OB2 and Taurus, Maddalena et al. (1986) for Orion A and B, Liseau et al. (1992) for Vela, and de Geus, Bronfman, and Thaddeus (1990) for $\rho$-Ophiucus. 2) Total number of the selected sources with $f_{60}>f_{12}$ and good or moderate flux quality at $60 \mu \mathrm{m}, \mathrm{FQ}_{60} \geq 2$; these are classified into four groups based on the flux quality at 12 and $100 \mu \mathrm{m}$, i.e. (FQ100, $\left.F Q_{12}\right)=(\geq 2, \geq 2),(\geq 2,1),(1, \geq 2)$, and $(1,1)$. The numbers of the sources for four groups are denoted in parenthesis. 
each star-forming region within the area where there is a single molecular cloud in the line of sight (Sargent 1979; Dame et al. 1987; Maddalena et al. 1986; Ungerechts \& Thaddeus 1987; Wouterloot \& Brand 1989; de Geus, Bronfman \& Thaddeus 1990).

We list the data of the individual IRAS point sources in Table 2. The IRAS point sources are divided into two groups in each star-forming region by the flux quality at $100 \mu \mathrm{m}, \mathrm{FQ} Q_{100}$; the first group is for $F Q_{100} \geq 2$ and the second for $F Q_{100}=1$. In the present paper we use mainly the data of the first group. The data of the second group are listed for more complete study in future. All of the IRAS data are taken from IRAS $P S C$. The parameters in Table 2 are as follows:

Column 1: IRAS name.

Columns 2 and 3 : Galactic coordinate which is transformed from the equatorial coordinate of IRAS PSC.

PER-OB2

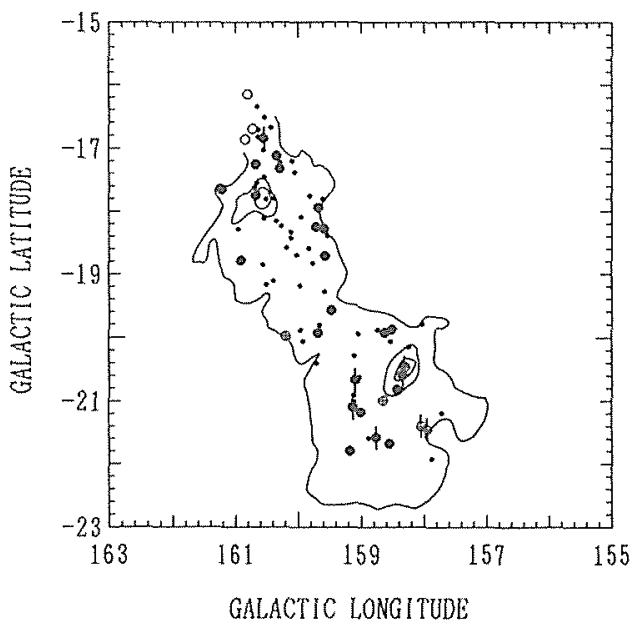

ORION-A

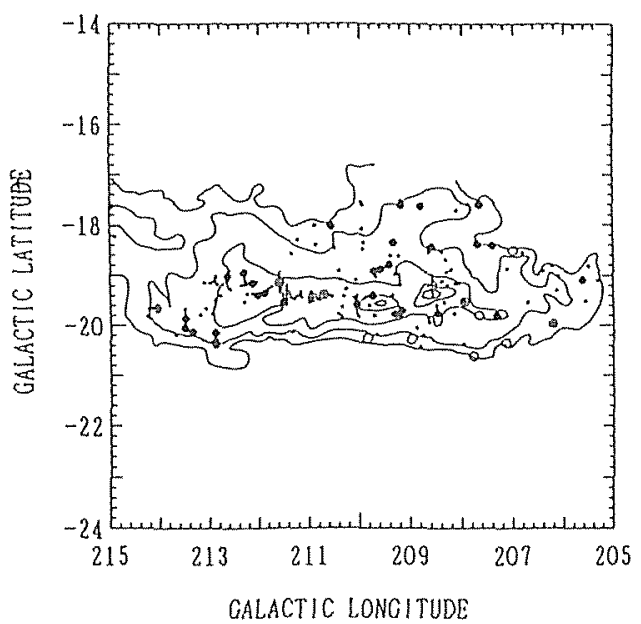

TAURUSS

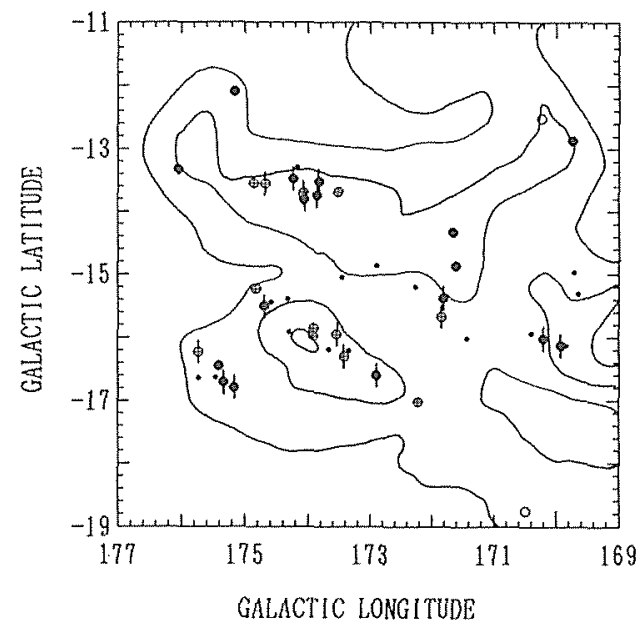

ORION-B

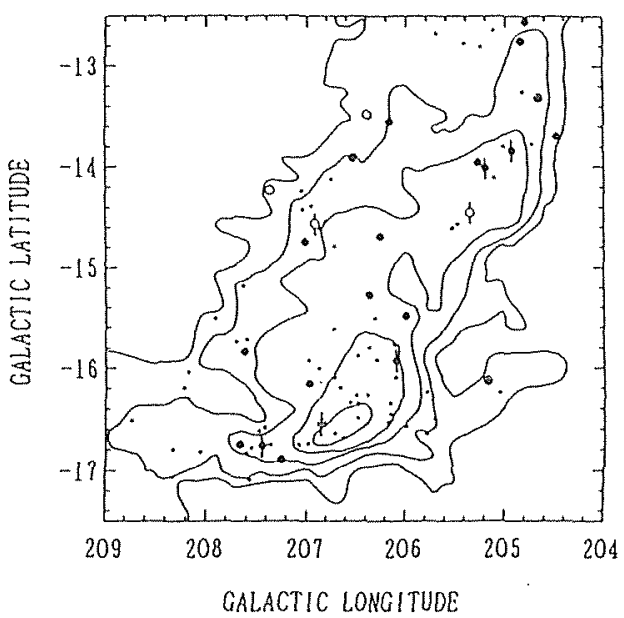

Fig. 2 

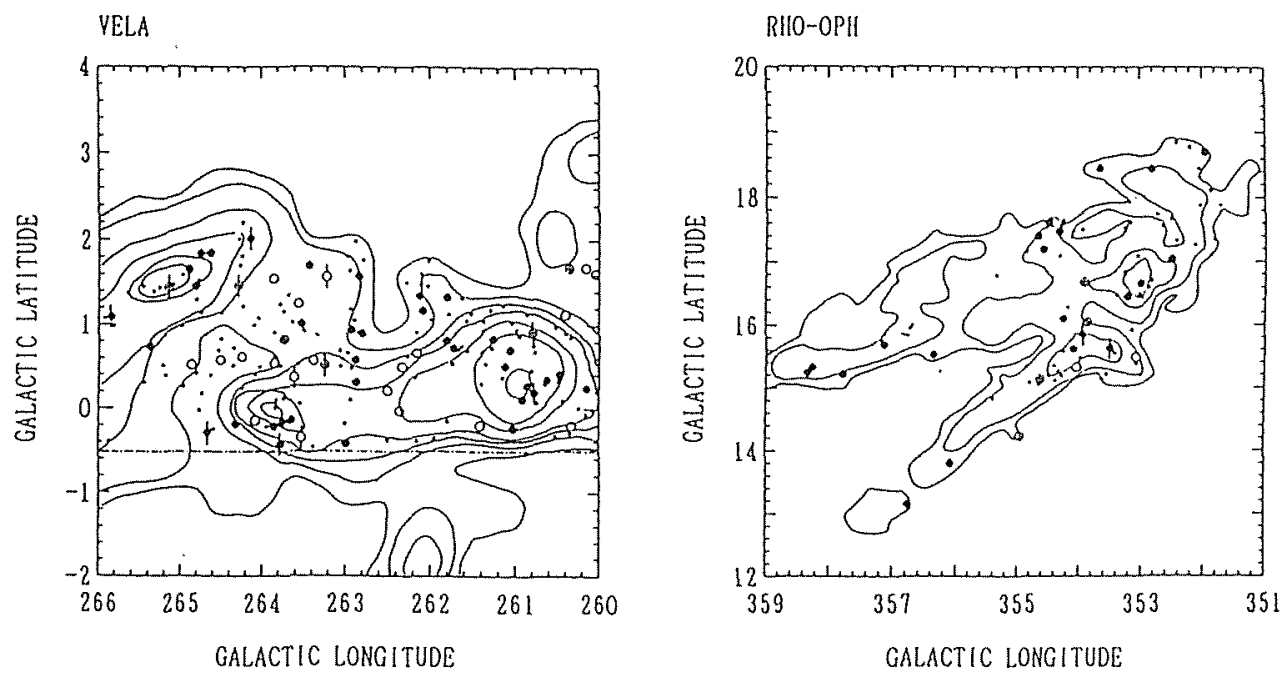

Fig. 2. Sky distribution of all of the selected IRAS point sources (i.e., cold YSOs) in the six nearby star-forming clouds. The filled circles are invisible sources, the circles with cross are sources with visible stars, and the open circles are sources whose optical counterparts are uncertain; all of these sources have reliable flux densities at 60 and 100 $\mu \mathrm{m}$. The small dots are sources with upper limits of the flux densities at $100 \mu \mathrm{m}$, and are not used in the FIR $H-R$ diagrams of Figure 3. The vertical bars indicate that the sources show some active features such as molecular outflow, $\mathrm{H}_{2} \mathrm{O}$ maser, and $\mathrm{HII}$ region. The contours indicate the ${ }^{12} \mathrm{CO}$ intensity following Sargent (1979) for PerOB2, Dame et al. (1987) for Taurus and Vela, Maddalena et al. (1986) for Orion A and $B$, and de Geus et al. (1990) for $\rho$-Ophiuchus. The outermost contours indicate the antenna temperature of $2.5 \mathrm{~K}$ for Per-OB2, the intensity of $5 \mathrm{~K} \mathrm{~km} \mathrm{~s}^{-1}$ for Taurus, Vela and $\rho$-Ophiuchus, and $1.28 \mathrm{~K} \mathrm{~km} \mathrm{~s}^{-1}$ for Orion $\mathrm{A}$ and $\mathrm{B}$.

Column 4: Flux qualities (FQ) for IRAS four bands in order of $12,25,60$, and 100 $\mu \mathrm{m} ; 1=$ upper limit, $2=$ moderate quality, and $3=$ high quality. The values of columns 5 to 10 are obtained even in the cases of $F Q_{\lambda}=1$ by using the upper limit values.

Column 5 : Color [60-12] defined by $\log \left(f_{60} / f_{12}\right)$, where $f_{\lambda}$ is the color-corrected flux density at $\lambda \mu \mathrm{m}$ (IRAS Explanatory Supplement). We define cold IRAS point sources by this color so that the values are positive for the original values of $f_{12}$ and $f_{60}$ in IRAS PSC.

Column 6 : The upper is color $[100-60]$ defined by $\log \left(f_{100} / f_{60}\right)$, which is used as a parameter of the FIR H-R diagram. The color-corrected flux densities are used. The lower is dust (color) temperature derived by equation (Emerson $1988 \mathrm{a}$ ),

$$
T_{d}=\frac{41.66}{[100-60]+0.222(\beta+3)}
$$

for $\beta=1$. The value is denoted for the first group only.

Column 7: Uncertainty of the color [100-60], calculated from the uncertainties of $f_{60}$ and $f_{100}$. This column is blank for the second group.

Column 8: The upper is logarithm of the luminosity at $60 \mu \mathrm{m}$ defined by $L_{60}=d^{2} f_{60}$, where $d$ is distance in kpc to the star forming region (see Table 1 ) and $f_{60}$ is the color-corrected flux density in Jy. $\log L_{60}$ is related with logarithm of the 


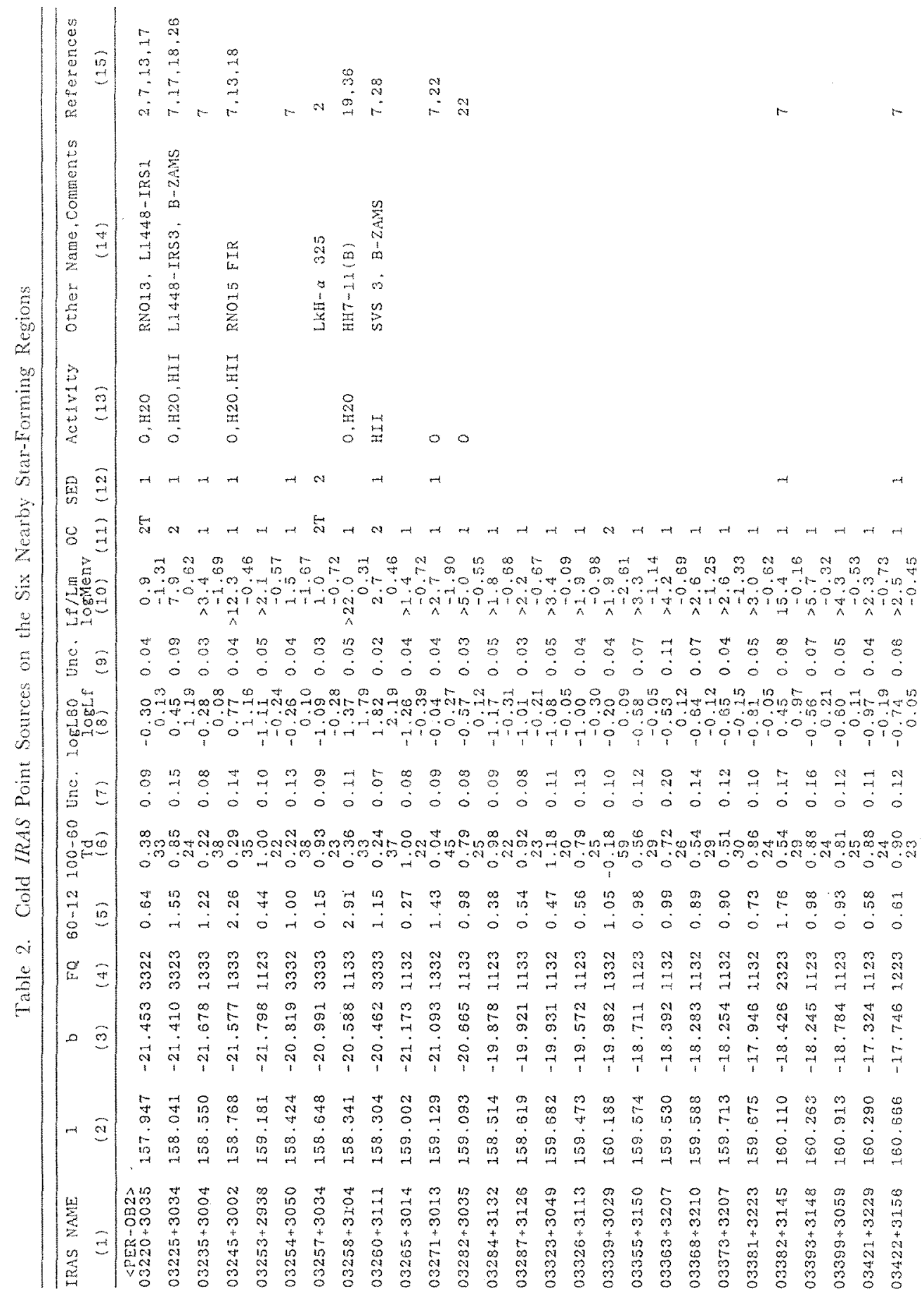




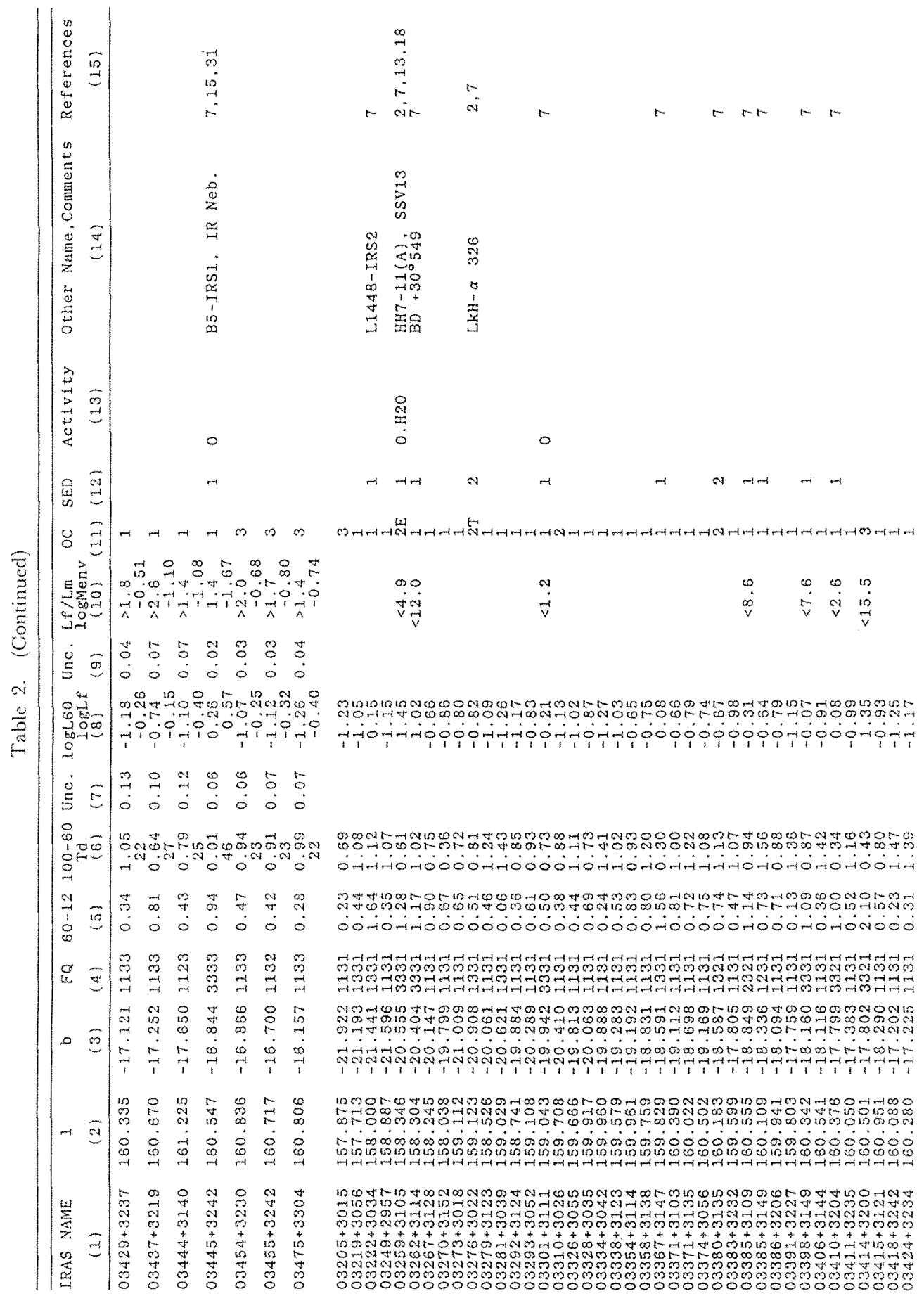




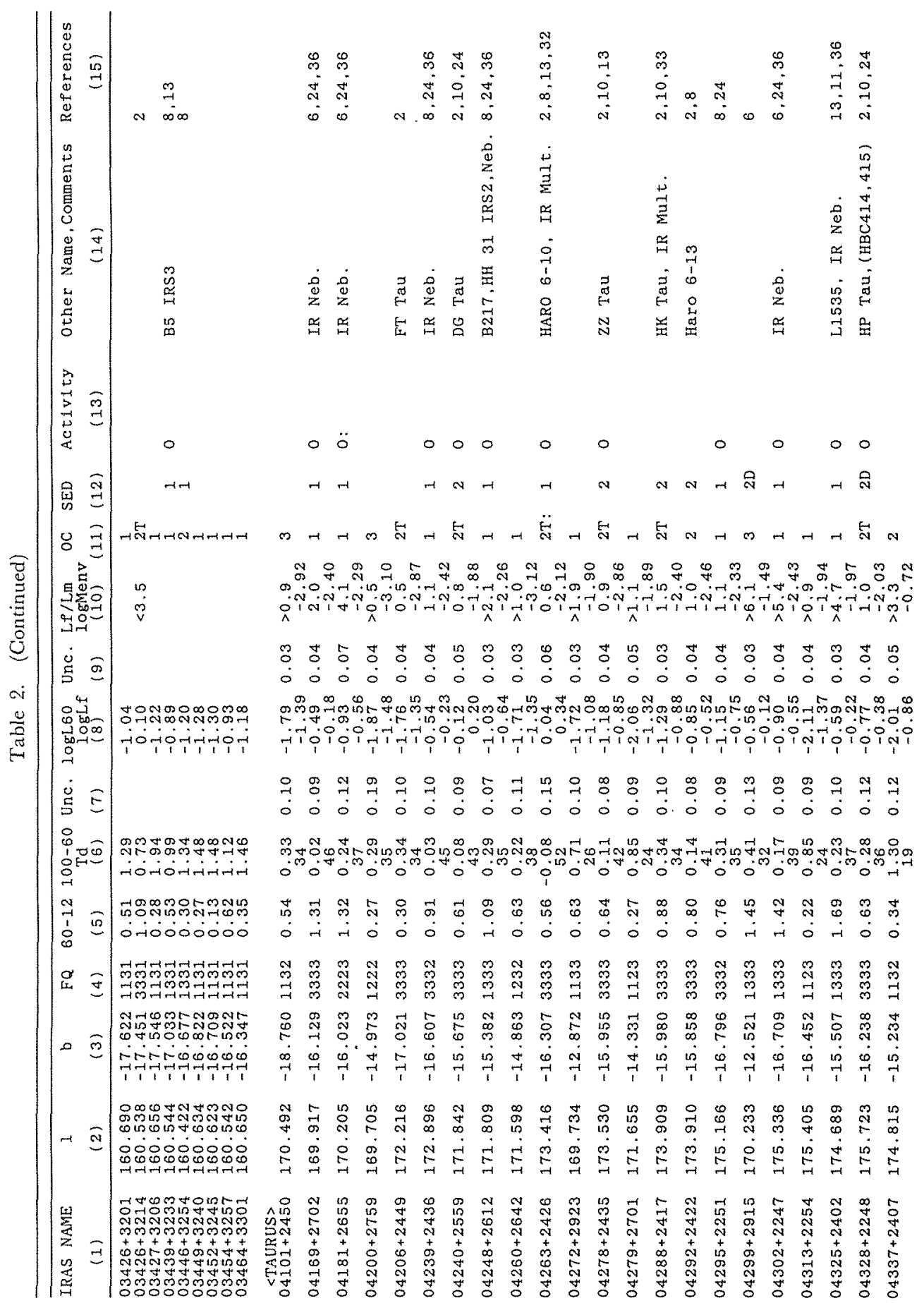




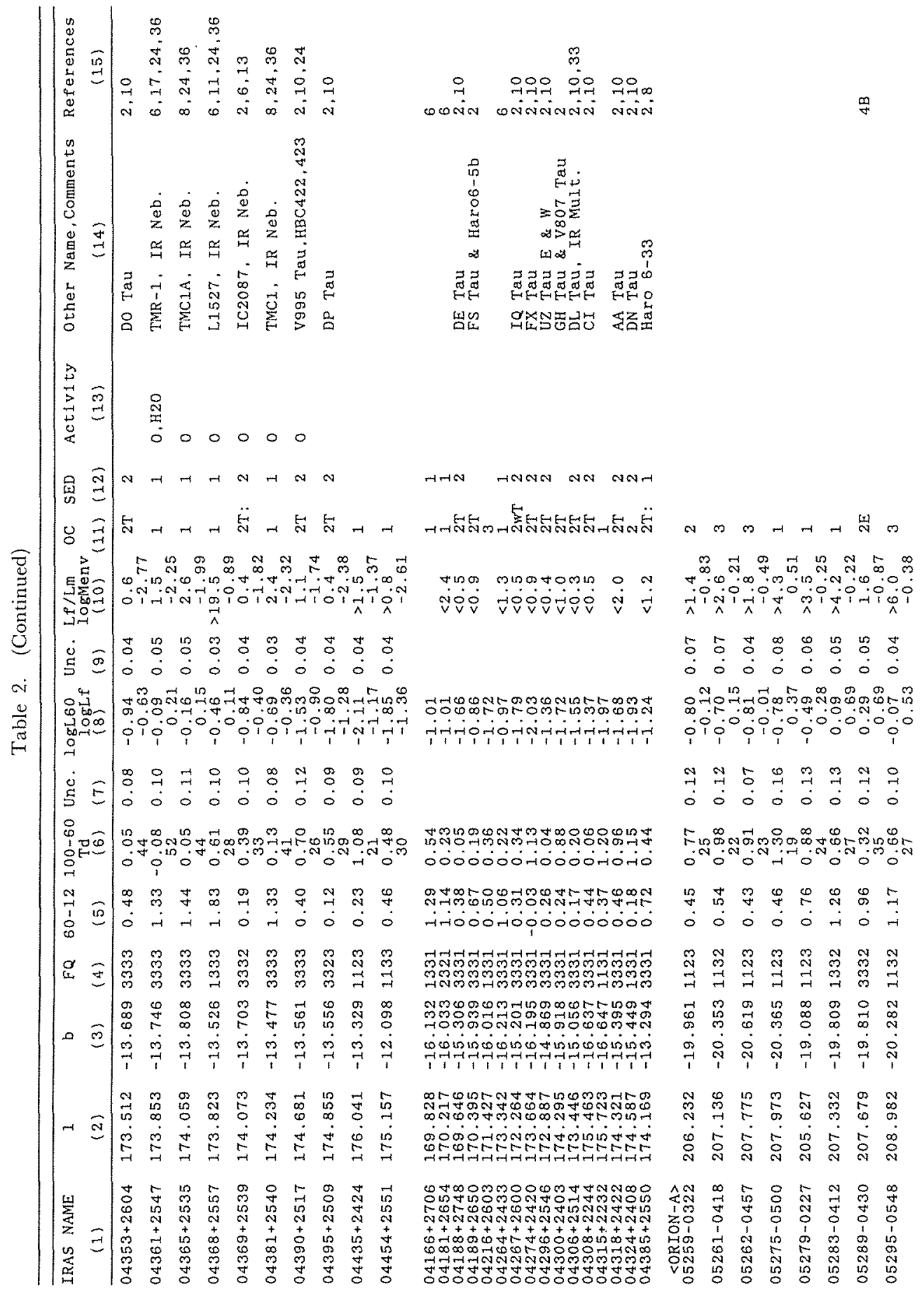




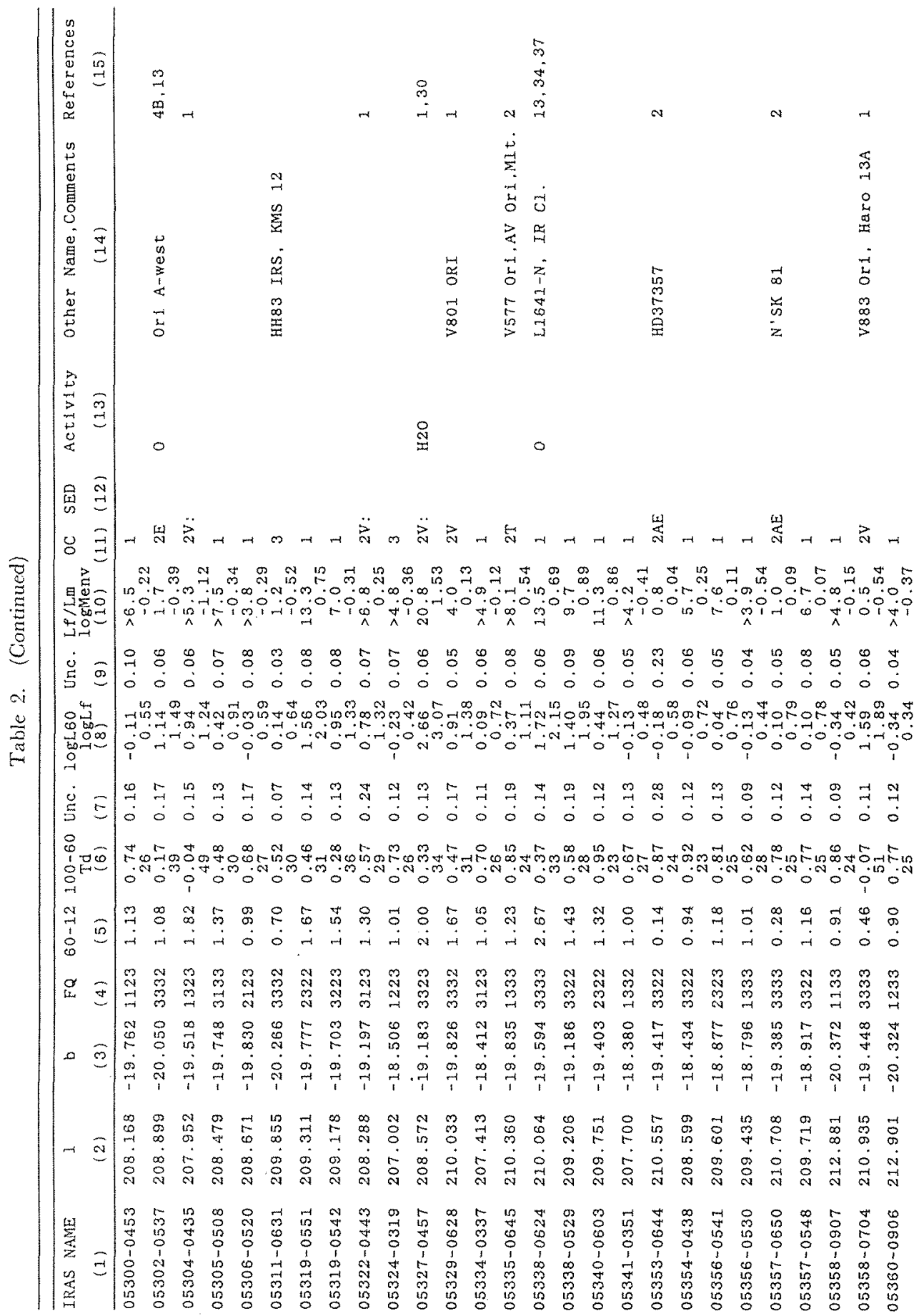




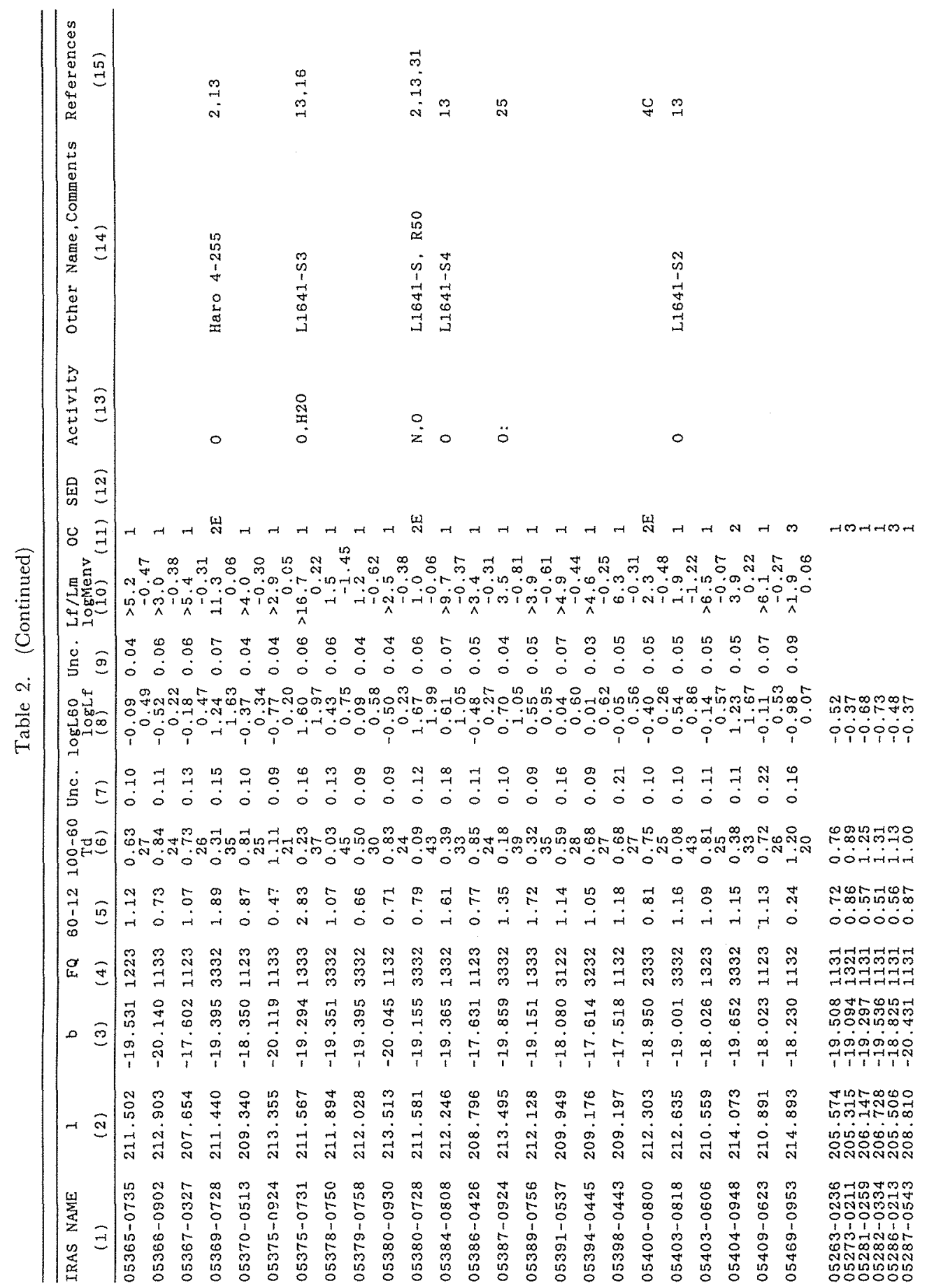




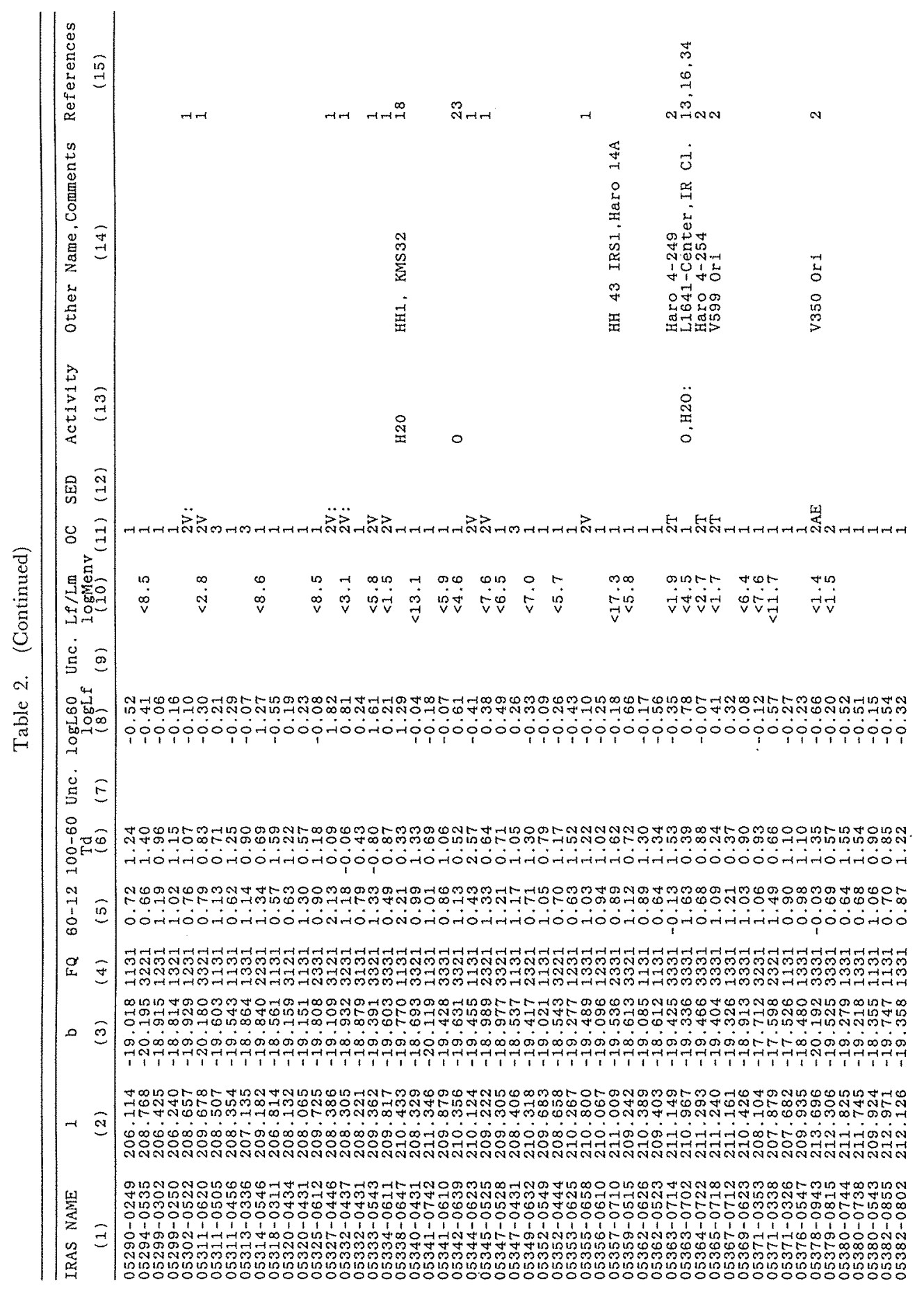




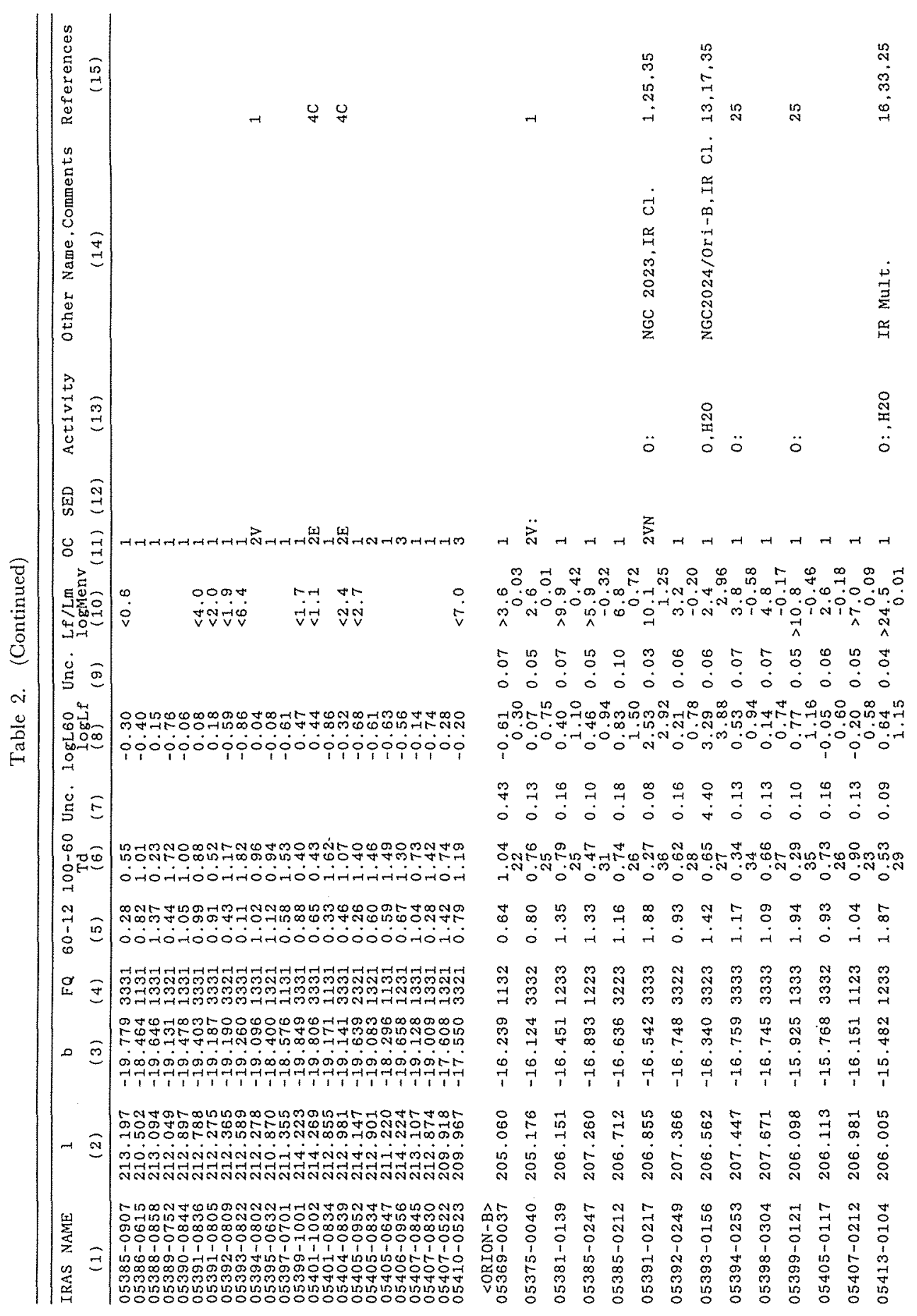




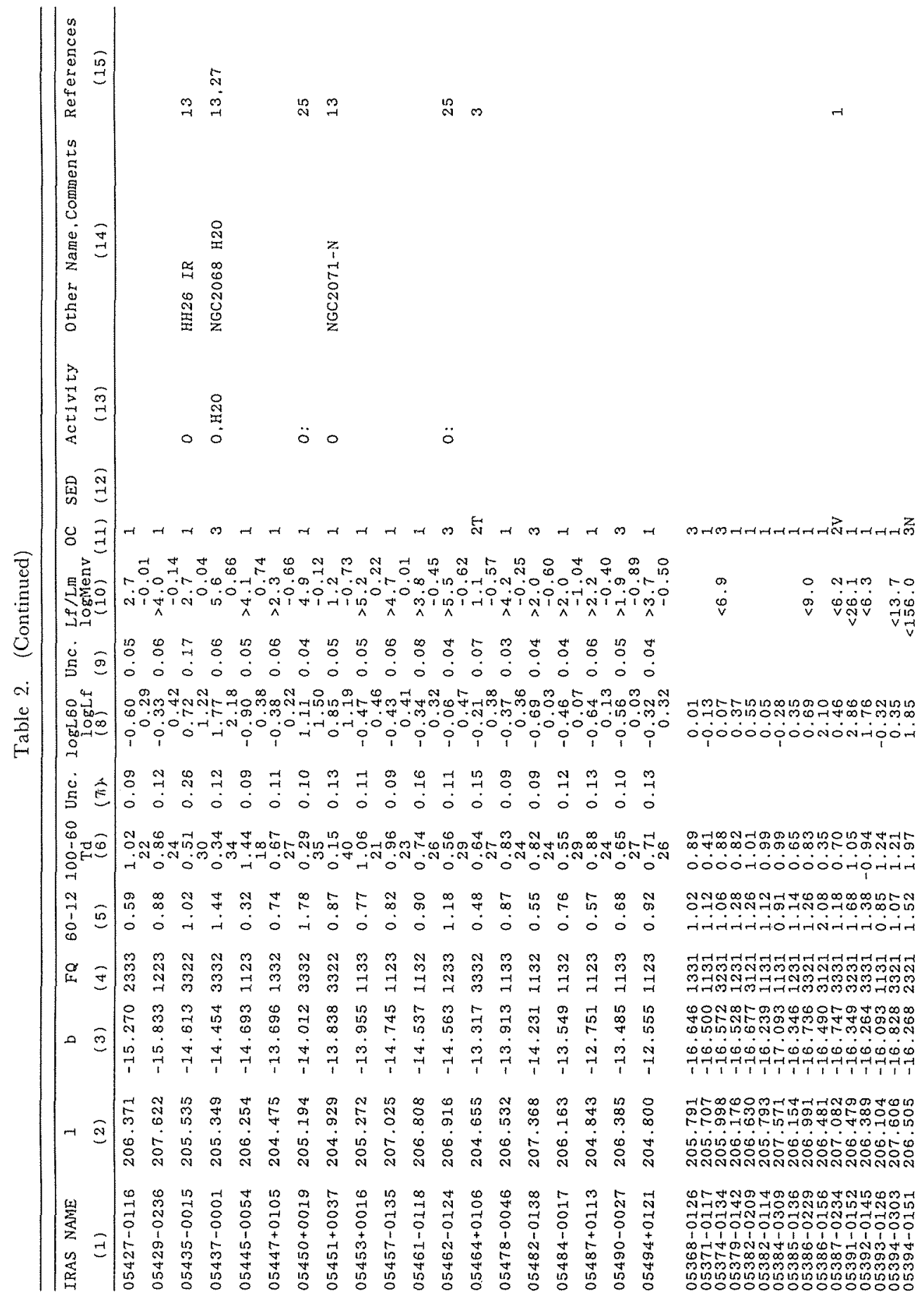




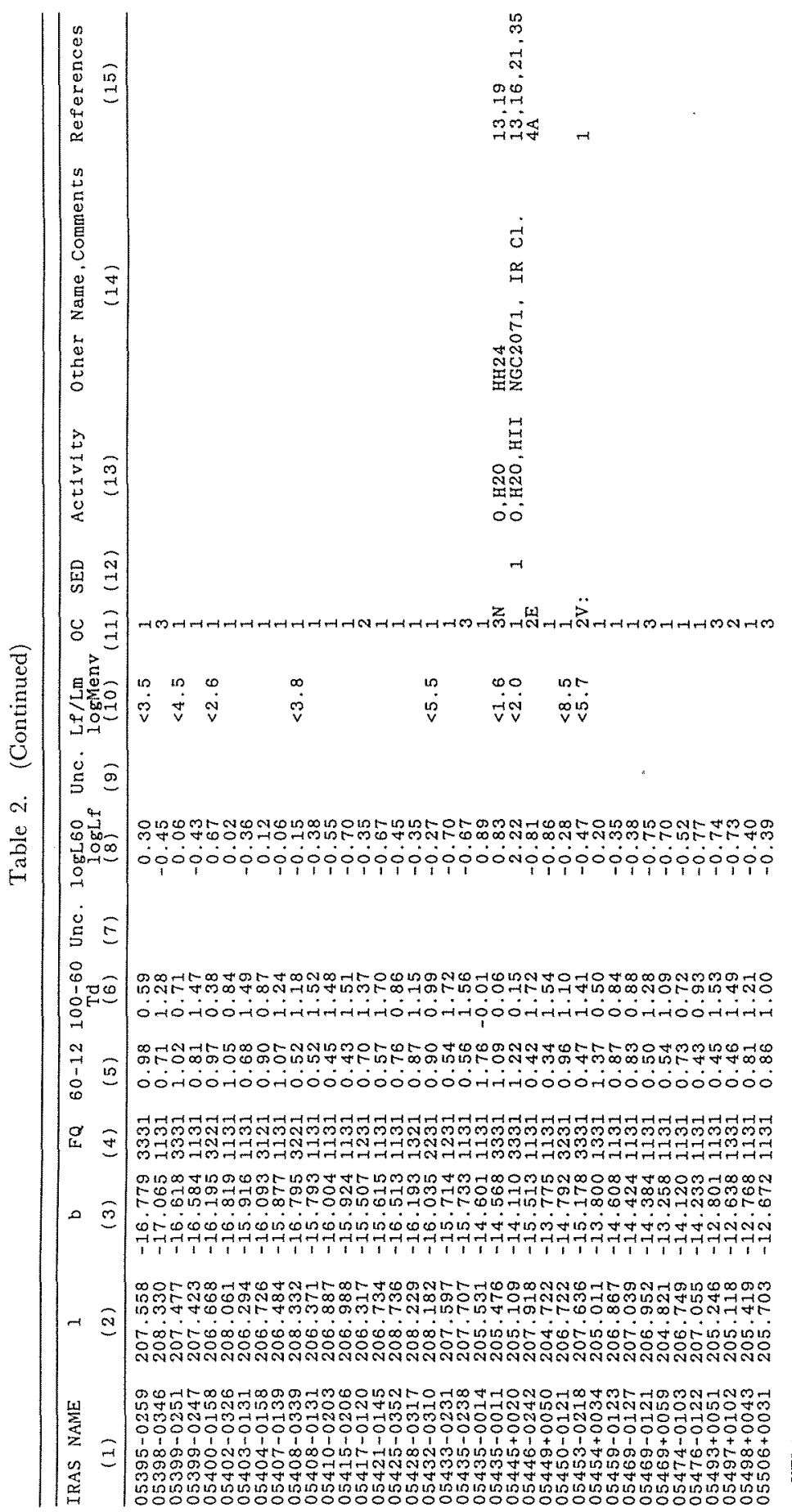
Nio. $\because$ :

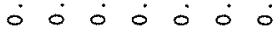
लำ

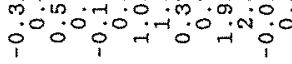
각 욱 겅 $\therefore \quad 0 \quad 0 \quad 0 \quad 0 \quad 0 \quad 0$

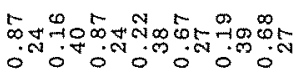

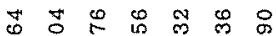
० $-\dot{0}-i-i \quad \dot{0}$

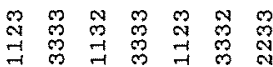
号 竎芯 i 0 i 婴

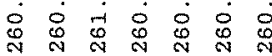

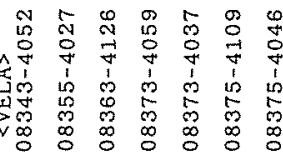




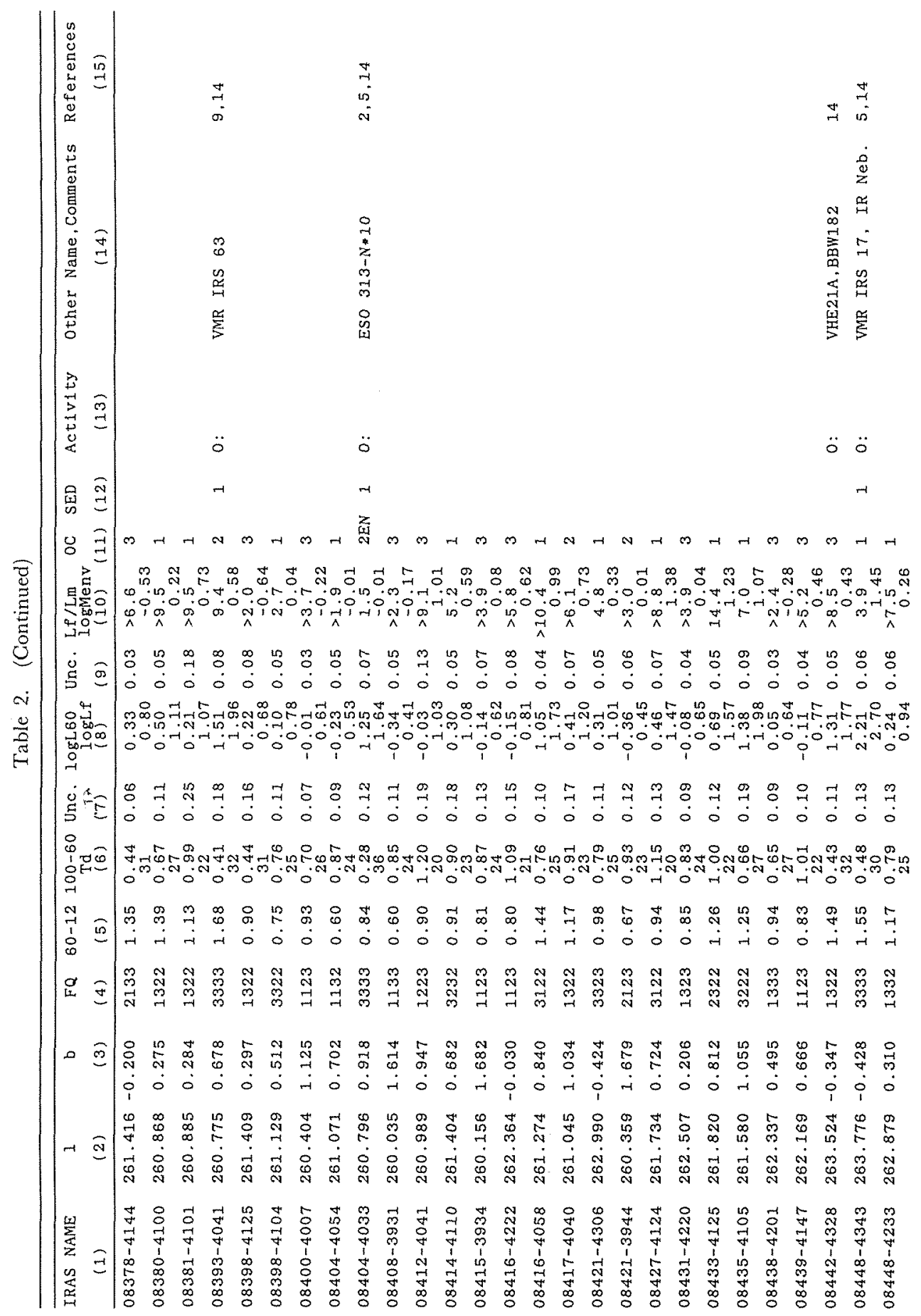




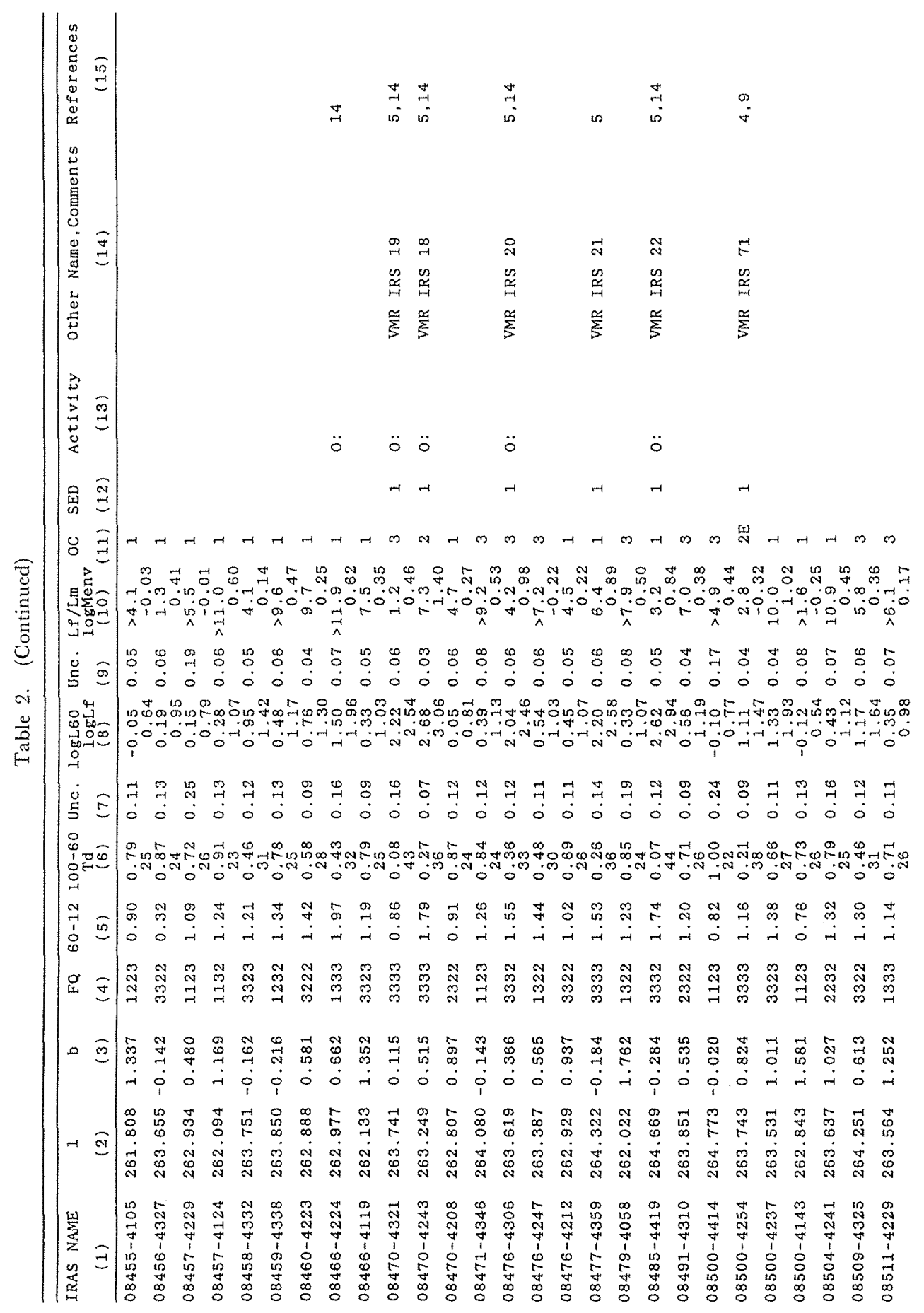




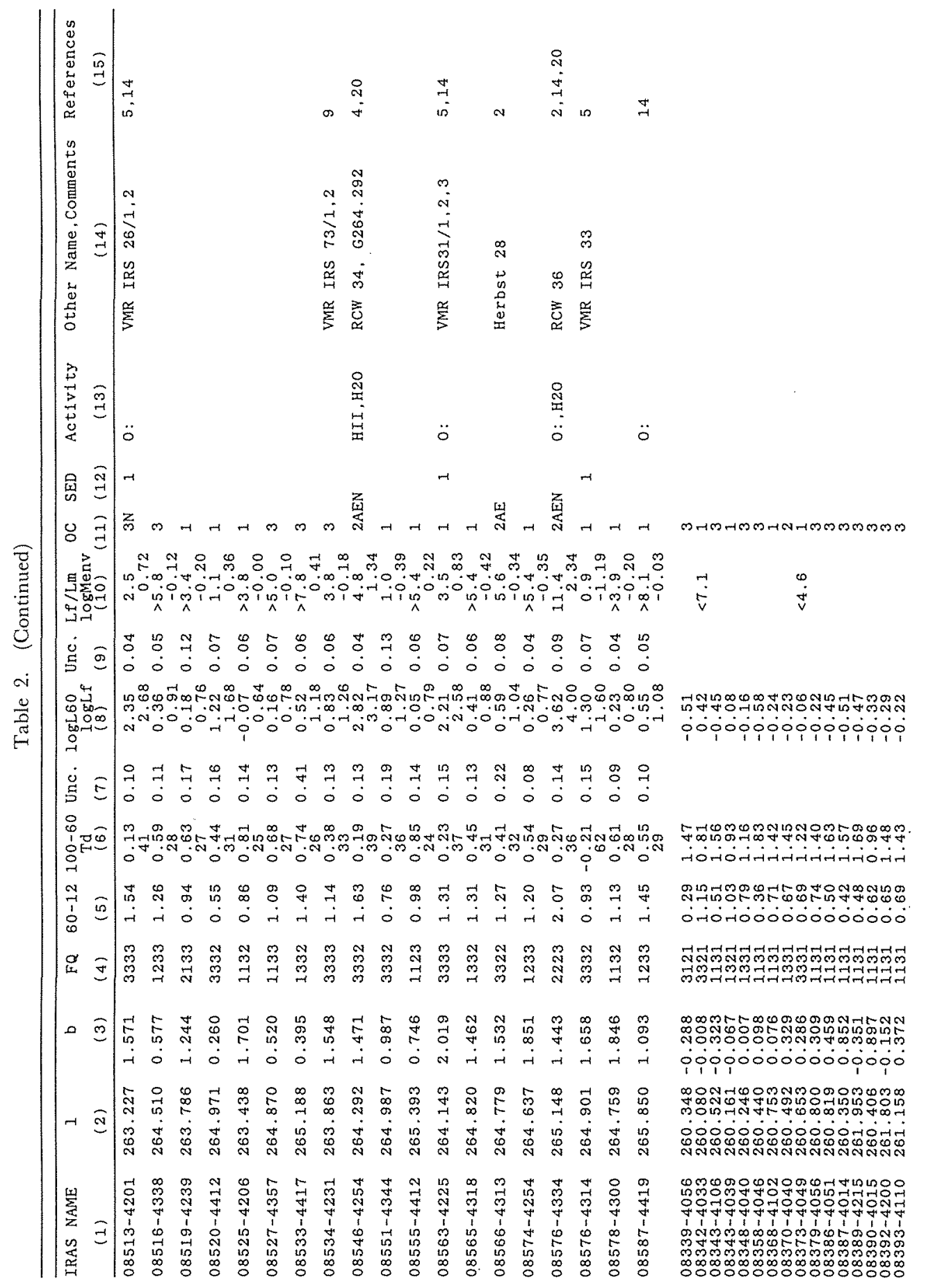




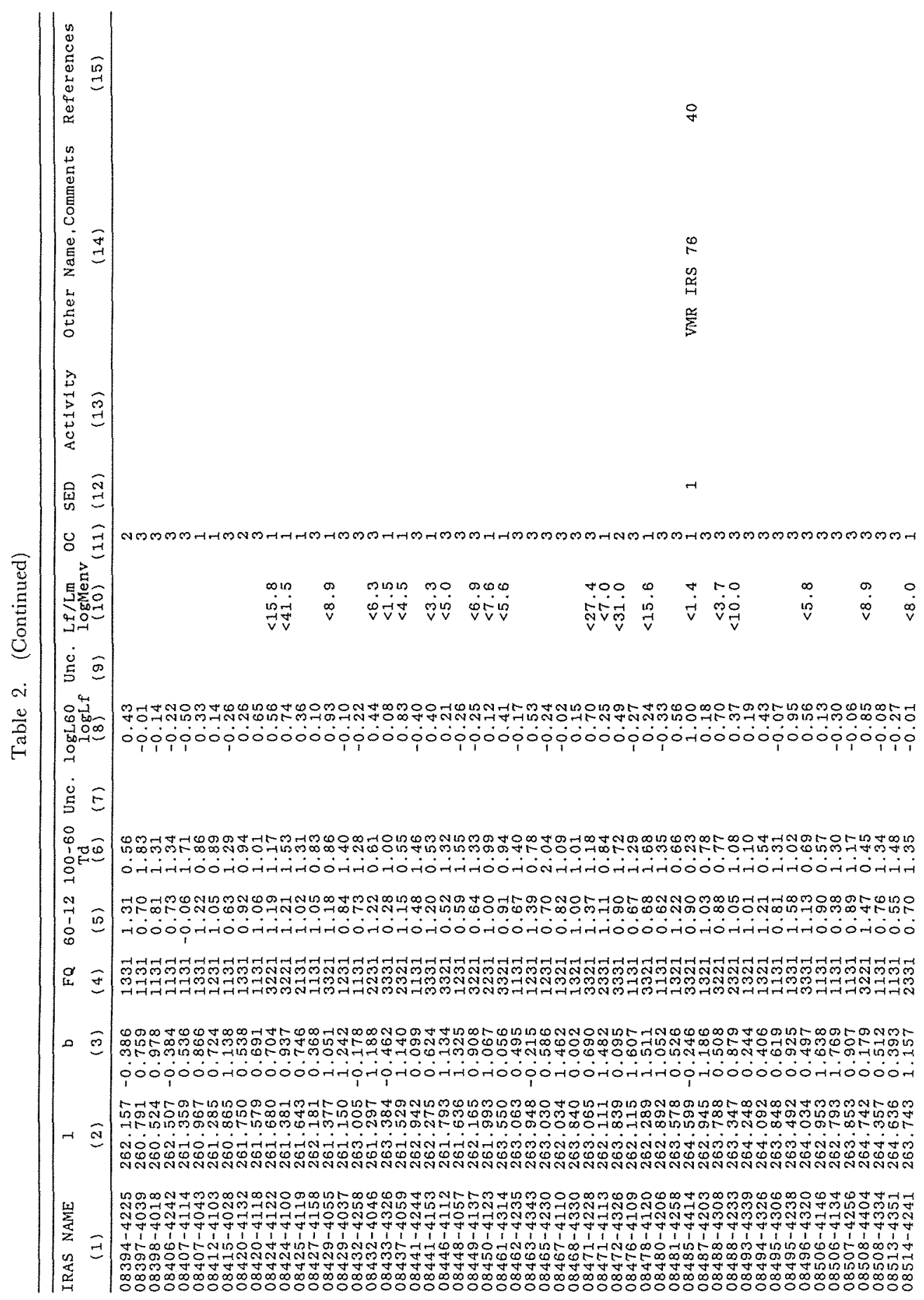




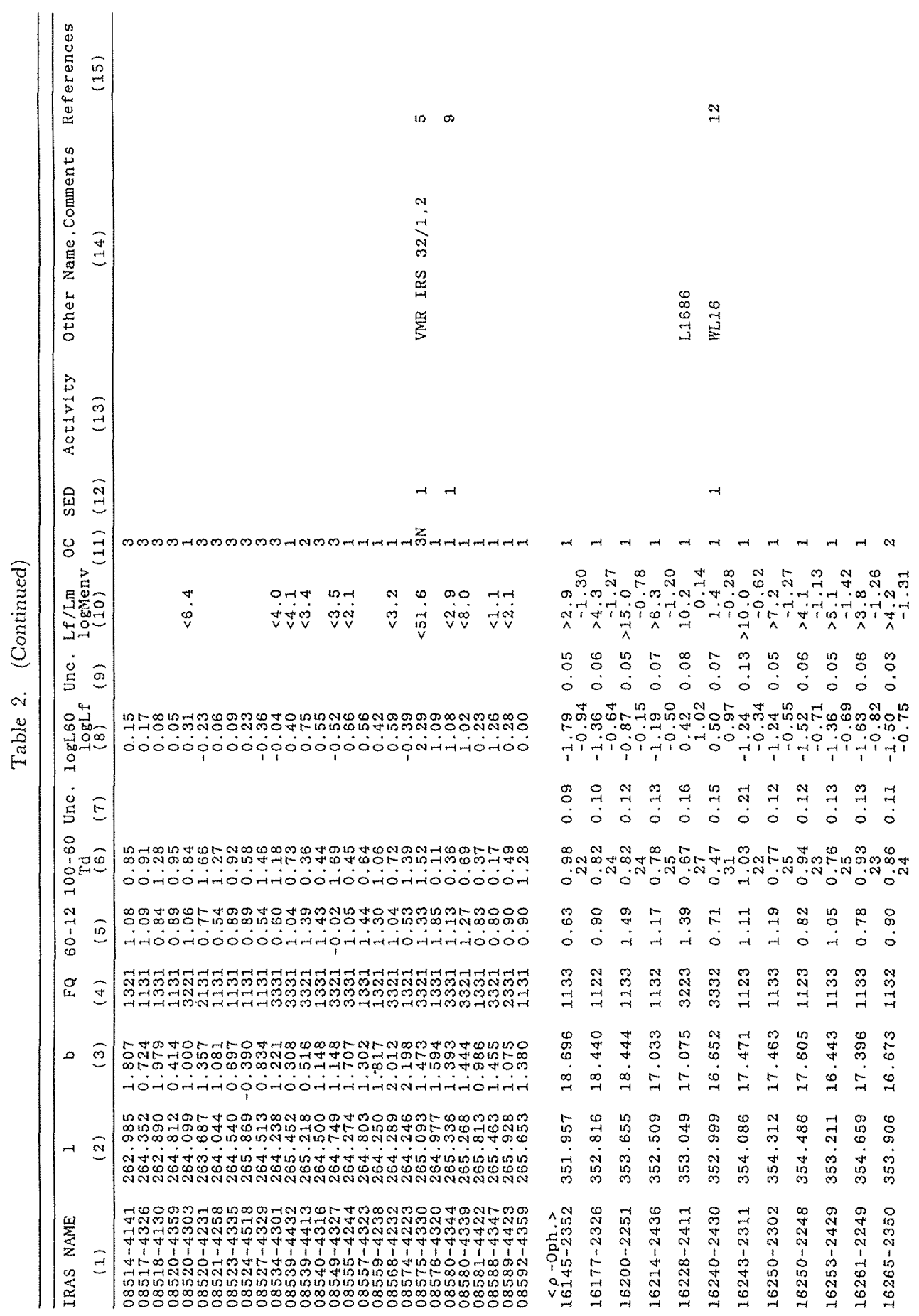




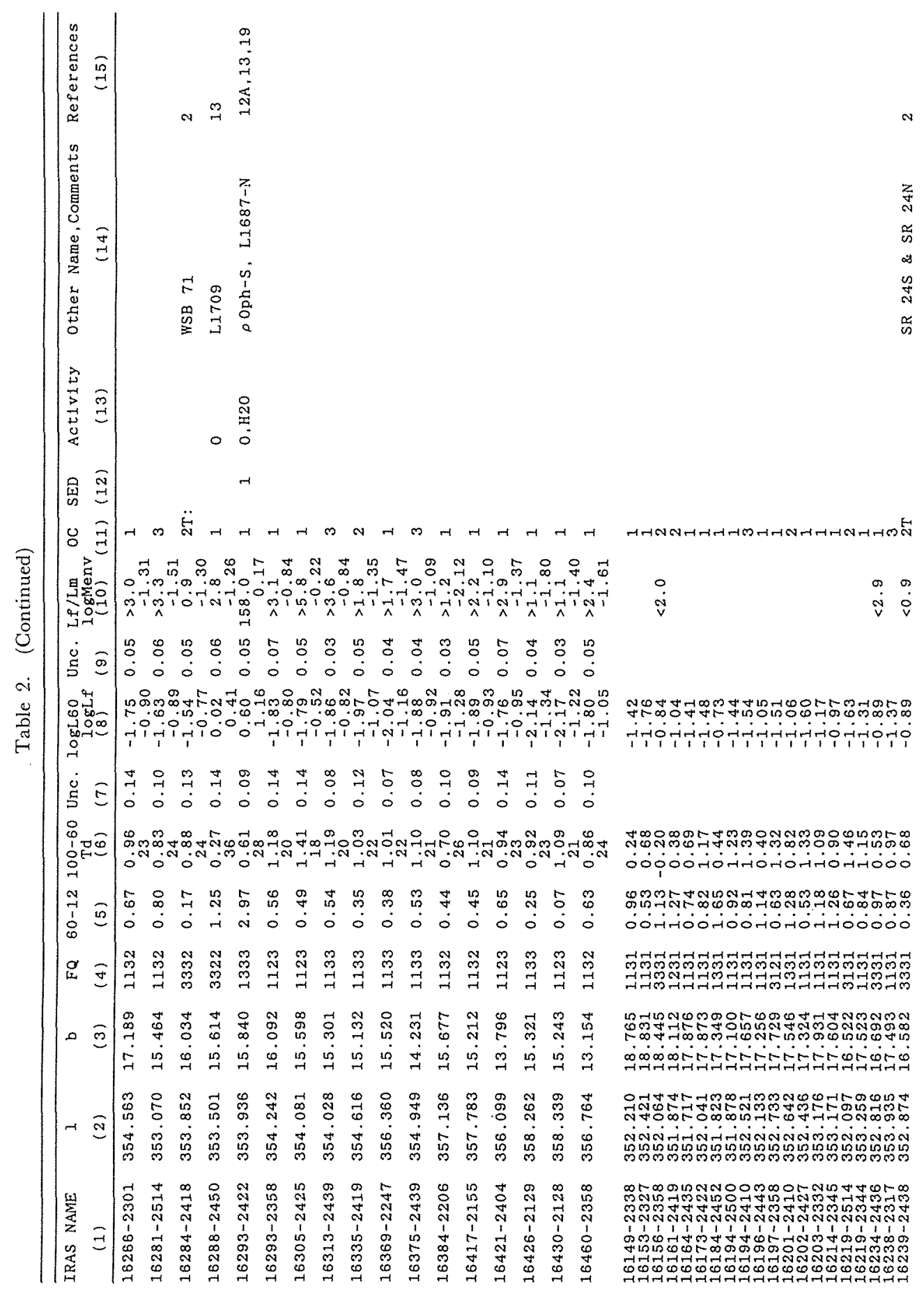



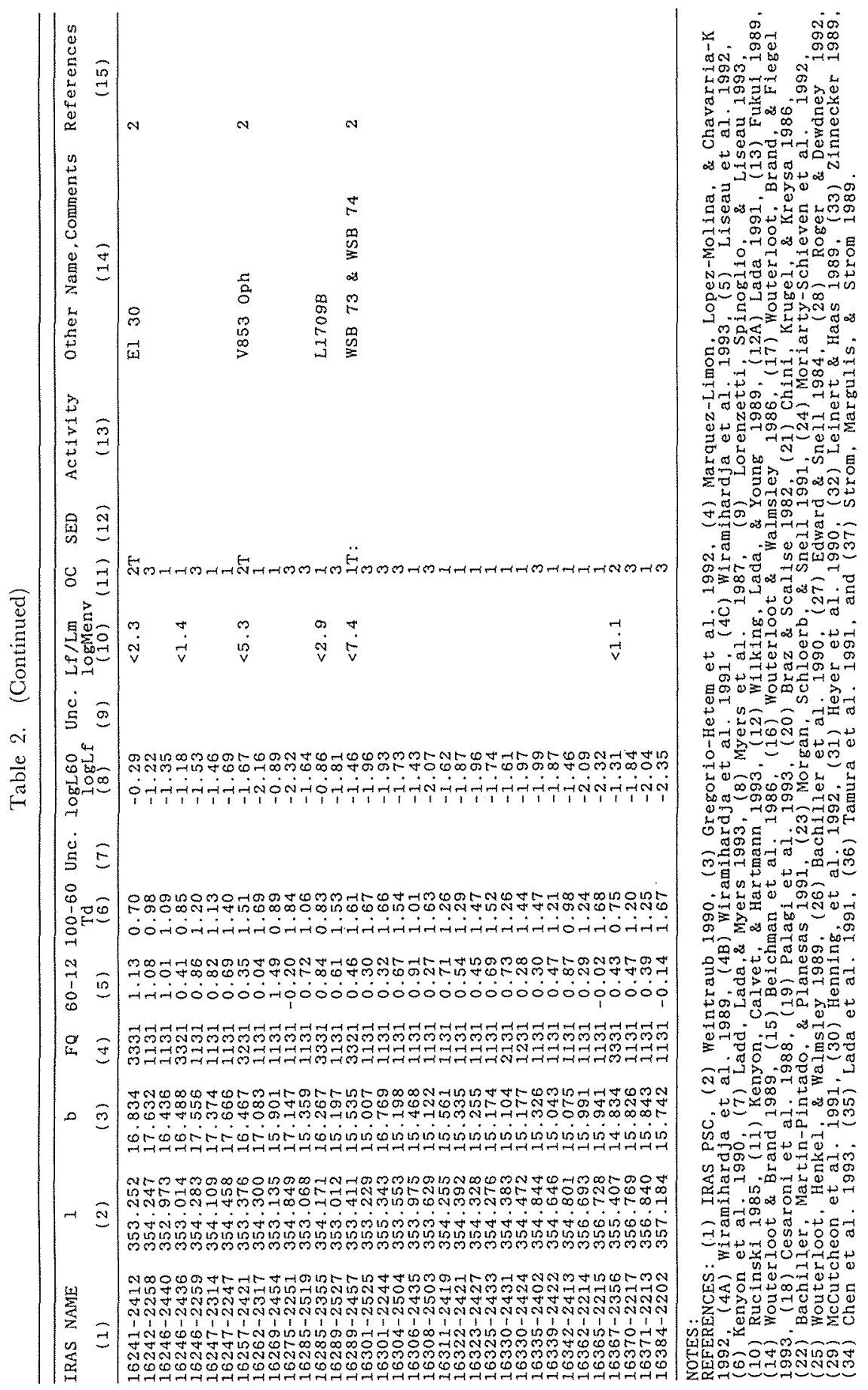
luminosity at $30-75 \mu \mathrm{m}$ as $\log \left(L_{30-75} / L_{\odot}\right)=\log L_{60}+0.15$ (Emerson $1988 \mathrm{~b}$ ). $\quad \log L_{60}$ is another parameter of the FIR H-R diagram. The lower is logarithm of the FIR luminosity in solar unit obtained by equation (Emerson $1988 \mathrm{~b}$ ),

$$
L_{f}=0.31 d^{2}\left(4.578 f_{60}+1.762 f_{100}\right) L_{\odot} .
$$

The value is denoted for the first group only.

Column 9: Uncertainty of $\log L_{60}$, obtained from the uncertainty of $f_{60}$. This column is blank for the second group.

Column 10: The upper is the luminosity ratio of FIR (30 to $135 \mu \mathrm{m}$ ) to midinfrared $(7$ to $30 \mu \mathrm{m})$ given by Emerson $(1988 \mathrm{~b})$, i.e.,

$$
L_{f} / L_{m}=\frac{\left(4.578 f_{60}+1.762 f_{100}\right)}{\left(20.653 f_{12}+7.538 f_{25}\right)} \text {. }
$$

For the first group, the lower limits are shown for the sources with $\mathrm{FQ}_{12}=1$ and/or $\mathrm{FQ}_{25}=1$, while for the second group the upper limits are shown only for the sources with $F Q_{12} \geq 2$ and $\mathrm{FQ}_{25} \geq 2$. The $L_{f} / L_{m}$ features are indicated in the left panels of Figure 3. The lower is logarithm of mass of the FIR emitting envelope in solar unit. The value is assumed to be 100 times dust mass obtained by equation (5) in $\$ 2.3$ for $\beta=1$. The value for $\beta=2$ is 1.7 times the value for $\beta=1$. The value is denoted for the first group only.

Golumn 11: Optical image taken by our inspection and optical counterpart taken from literature; $1=$ invisible, $2=$ visible star, and $3=$ uncertain because there is a very faint star or some field stars or a bright nebula within 1' of the position of the IRAS point source. These features are indicated in Figure 2 and the right panels of Figure 3 . The meanings of the additional letters are $\mathrm{AE}=$ Herbig $\mathrm{Ae} / \mathrm{Be}$ star, $\mathrm{E}=$ emission line star, $\mathrm{N}=$ bright nebula, $\mathrm{T}=\mathrm{T}$ Tauri star, $\mathrm{V}=$ variable star, and $\mathrm{w} \mathrm{T}=$ weak line $\mathrm{T} \mathrm{T}$ auri star. The colon means probable feature.

Column 12: Infrared SED Glass taken from literature ; $1=$ Class I, $2=$ Class II, and $2 \mathrm{D}=$ Class IID.

Column 13: Active feature taken from literature; $\mathrm{H} \mathrm{II}=\mathrm{H}$ II region, $\mathrm{H}_{2} \mathrm{O}=\mathrm{H}_{2} \mathrm{O}$ maser, $\mathrm{O}=\mathrm{CO}$ outflow, and $\mathrm{O}:=\mathrm{CO}$ wing or broad $\mathrm{CO}$ line. The features are indicated in Figure 2 and the right panels of Figure 3.

Column 14: Other name and comment. The comments are IR Neb.=infrared nebulosity, IR CI.=cluster of infrared stars, Mult. = two or more stars are associated with the IRAS point source, and $\mathrm{B}-\mathrm{ZAMS}=\mathrm{B}$-type zero age main sequence star.

Column 15: Reference for the features cited in Columns 11 to 14 (see footnote of Table 2).

\subsection{FIR H-R Diagrams}

We construct a FIR H-R diagram of YSOs in each star-forming region. The abscissa is the color $\log \left(f_{100} / f_{60}\right) \equiv[100-60]$ and the ordinate is logarithm of the luminosity at $60 \mu \mathrm{m}$ defined as $\log \left(d^{2} f_{60}\right) \equiv \log L_{60}$, the values of which are, respectively, listed in Columns 6 and 8 in Table 2.

The FIR H-R diagrams of the six star-forming regions are shown in Figure 3 , in which we plotted only the sources belonging to the first group in Table 2 (i.e., 

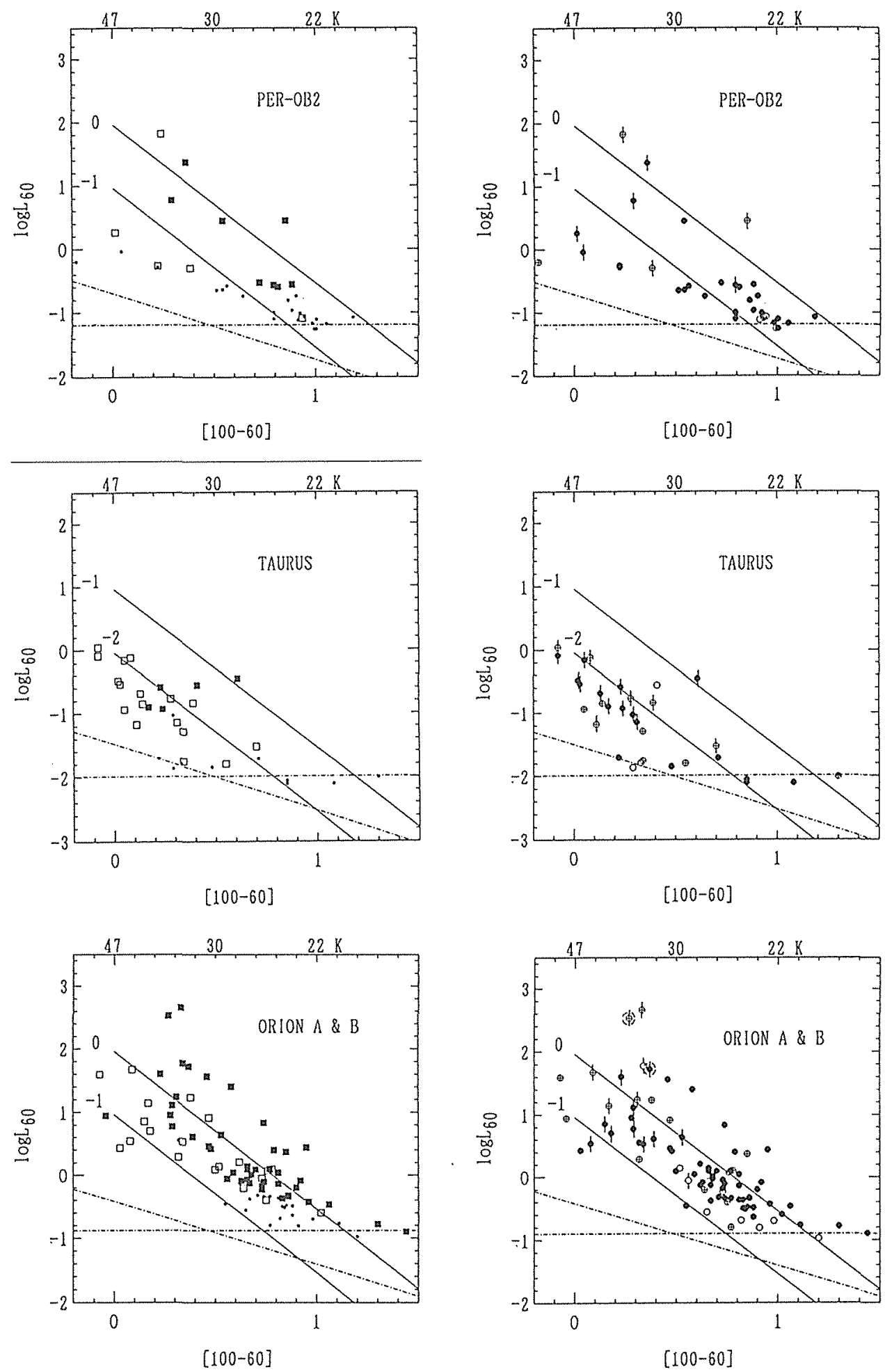

Fig. 3 


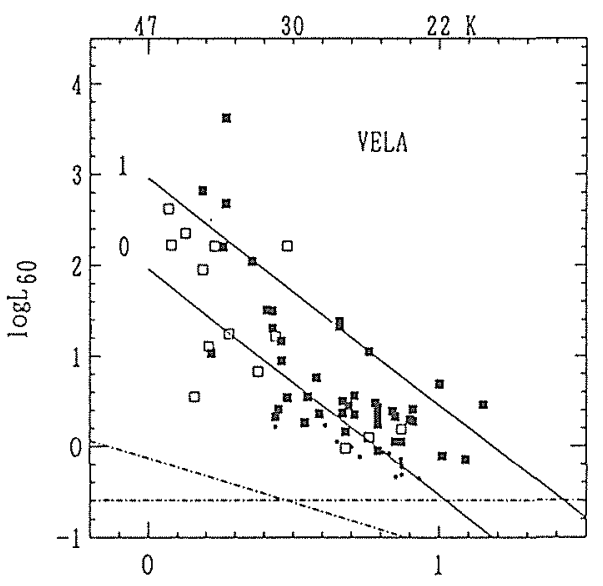

$[100-60]$

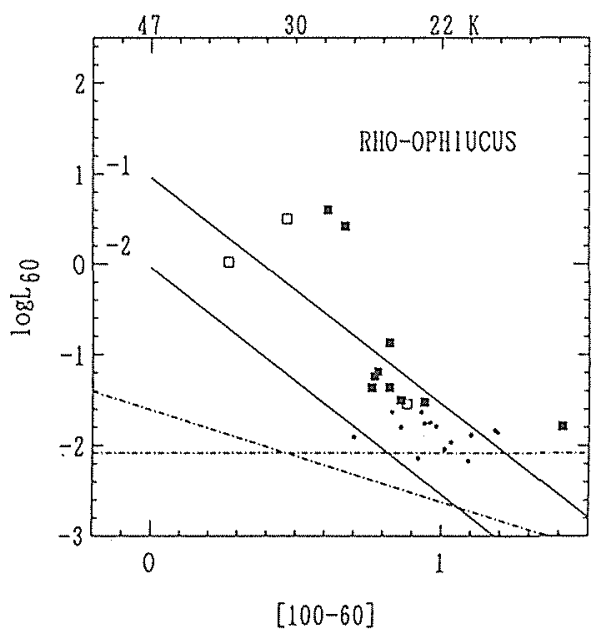

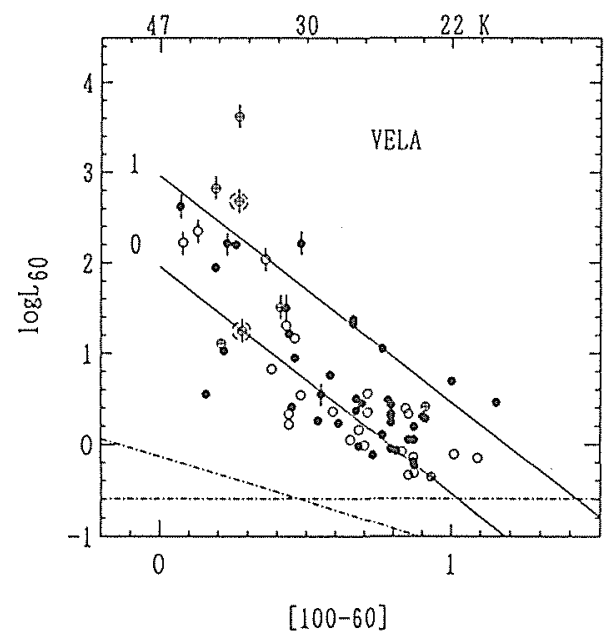

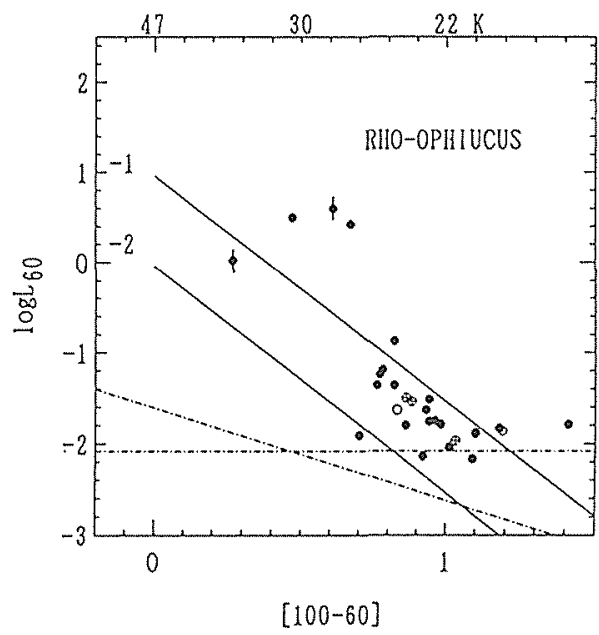

Fig. 3. FIR H-R diagrams of YSOs for the six star-forming regions at distance $d \mathrm{kpc}$; the ordinate is $\operatorname{logarithm}$ of the $60 \mu \mathrm{m}$ luminosity, $\log d^{2} f_{60} \equiv \log L_{60}$, and the abscissa is the FIR color, $\log \left(f_{100} / f_{60}\right) \equiv[100-60]$. The $L_{60}$ is related to the luminosity for 30 to $75 \mu \mathrm{m}$ (Emerson 1988b) as $\log \left(L_{30-75} / L_{\odot}\right)=\log L_{60}+0.15$. Orion $\mathrm{A}$ and $\mathrm{B}$ are combined in a diagram. The uncertainties are $\leq 0.20$ in $[100-60]$ and $\leq 0.10$ in $\log L_{60}$. The left panels: The symbols show the luminosity ratio of FIR to mid-infrared, $L_{f} / L_{m}$; the filled squares are the YSOs with $L_{f} / L_{m}>4$ (the extreme Class I YSOs), the open squares are the YSOs with $L_{f} / L_{m}<4$, and the dots are the YSOs whose values of $L_{f} / L_{m}$ are uncertain wheter the values are larger than 4 . The horizontal dash-dotted line in each diagram represents $f_{60}=0.5 \mathrm{Jy}$, and the inclined line $f_{100}=1.5 \mathrm{Jy}$, which are the IRAS sensitivity limits. The solid lines show the constant envelope mass lines and the numbers shown are logarithm of the envelope mass in solar unit (see text). The right panels: The same as the left panels, except for the symbols. The filled circles are invisible YSOs, the circles with cross are the YSOs with visible stars, and the open circles indicate the YSOs whose optical counterparts are uncertain. The vertical bars indicate that the objects show the active features such as molecular outflow, $\mathrm{H}$ II region, and $\mathrm{H}_{2} \mathrm{O}$ maser. The bigdashed circles show the objects associated with infrared cluster. 
$\mathrm{FQ}_{100} \geq 2$ ) and having the uncertainties $\leq 0.20$ in $[100-60]$ and $\leq 0.10$ in $\log L_{60}$ (see Table 2). The YSOs excluded by the uncertainty criteria are 16 in total $(6 \%$ of the total of the first groups). The error bars in Figure 3 (not indicated) correspond to their mean uncertainties; these are 0.12 in the abscissa and 0.05 in the ordinate. Since the FIR H-R diagrams of Orion $A$ and $B$ are similar to each other, we combine them in a diagram. The color temperatures for $\beta=1$ are indicated on the upper abscissa. The temperature scale for $\beta=2$ shifts by -0.22 in the abscissa. The horizontal dash-dotted lines represent $f_{60}=0.5 \mathrm{Jy}$ and the inclined ones represent $f_{100}=1.5 \mathrm{Jy}$, which are, respectively, the sensitivity limits of the IRAS measurements (IRAS Explanatory Supplement).

In the left panels of Figure 3, the symbols of plots indicate the luminosity ratio $L_{f} / L_{m}$ between the FIR and mid-infrared listed in Columm 10 of Table 2. The filled squares are the YSOs with $L_{f} / L_{m}>4$. In this paper we call those the extreme Class I YSOs, because the YSOs with the spectral index $\alpha=0$ have $L_{f} / L_{m} \simeq 1$ and they are still Class I objects. The extreme Class I YSOs are youngest among the selected YSOs. The open squares are the YSOs with $L_{f} / L_{m}<4$ and the dots indicate the YSOs whose values of $L_{f} / L_{m}$ are uncertain whether they are larger than 4 . In the right panels, the symbols show the optical counterparts and their activities, which are listed in Columns 11 and 13 of Table 2. The filled circles indicate invisible YSOs and the circles with cross are the YSOs associated with visible stars, while open circles indicate the YSOs whose visible features are "uncertain" in our inspection. These features are denoted in Column 11 of Table 2 as 1, 2, and 3, respectively. The associations of visible stars have been also taken from literature (e.g., Weintraub 1990 and others). The circles with vertical bars indicate the objects showing the active features such as molecular outflow (e.g., Fukui 1989), probable embedded outflow (e.g., Wouterloot \& Brand 1989), compact $\mathrm{H}$ II region, and $\mathrm{H}_{2} \mathrm{O}$ maser (e.g., Wouterloot \& Walmsley 1986 ; Palagi et al. 1993). A cross-correlation between the $L_{f} / L_{m}$ and optical visibility is shown in Table 3.

About half of the selected YSOs have $\mathrm{FQ}_{100}=1$, as shown in Tables 1 and 2. Figure 4 shows the FIR H-R diagram for all of the selected YSOs in Orion A and B, where the circles and crosses indicate the objects with $F Q_{100} \geq 2$ and $=1$, respectively. Most of the objects with $F Q_{100} \geq 2$ have the color $[100-60]=0$ to 1.2 , while the objects with $\mathrm{FQ}_{100}=1$ tend to have higher color values due to the upper limits of $f_{100}$. These trends also appear in the FIR H-R diagrams for the other four star-forming regions.

Table 3. Gorrelation between Optical Visibility and $L_{f} / L_{m}{ }^{1)}$

\begin{tabular}{lcccc}
\hline \multicolumn{1}{c}{$L_{f} / L_{m}$} & $>4$ & $<4$ & Uncertain & $<4^{2)}$ \\
\hline Invisible & 86 & 33 & 99 & 25 \\
Visible star & 16 & 25 & 12 & 24 \\
Uncertain & 21 & 5 & 32 & 5 \\
\hline
\end{tabular}

1) The YSOs, except for those in the last column, are all plotted in the FIR H-R diagrams of Figure 3. 2) The YSOs with $F Q_{100}=1$ in Table 2. 
Emerson (1987) deduces that cirrus confusion becomes severe for [100-60] $\geq 0.8$. But we found that the mean number of the cirrus flags (GIRRl) for the sources with $\mathrm{FQ}_{100}=1$ is not different from that of the sources with $F Q_{100} \geq 2$. We infer that the upper limits of $f_{100}$ are due to the relatively smaller flux densities of the sources. Chen, Tokunaga \& Fukui (1993) made IRAS co-added images in the Orion A region, the sensitivity of which is 2-3 times higher than that of IRAS PSC. They found reliable flux densities at $100 \mu \mathrm{m}$ for 14 IRAS point sources with $\mathrm{FQ}_{100}=1$ in Table 2, although their uncertainties are still relatively large. Such YSOs are shown on the FIR H-R diagram in Figure 5, on which the plots for the co-added data are located certainly on the permitted region for the YSOs with $F Q_{100} \geq 2$ in Figure 4. Thus, we consider that the sources with $F Q_{100} \geq 2$ shown in Figure 3 form a sample of cold, bright YSOs in each star-forming region.

Some brightest IRAS point sources in each star-forming region are often detected at the central part of infrared clusters, where the projected surface densities of YSOs are up to $\sim 100 \mathrm{pc}^{-2}$. In Orion A, Strom, Margulis \& Strom (1989) showed by a deep 2.2 $\mu \mathrm{m}$ imaging survey that IRAS 05338-0624 (L1641-N) contains 20 stellar or semi-stellar objects. IRAS PSC'S flag indicates that L1641-N is an extended source both in 12 and $25 \mu \mathrm{m}$. In Orion B, Lada et al. (1991 b) found many near-infrared sources in NGC 2023 (IRAS 05391-0217) and NGC 2024 (IRAS 05393-0156). NGC 2024 is not plotted on the FIR H-R diagram due to its large uncertainty in color [100-60]. Liseau et al. (1992) reported that in Vela, ESO $313-N^{*} 10$ (IRAS 08404-4033) is a cluster of 13 stars and VMR IRS 18 (IRAS 08470-4243) a cluster of three stars; the latter is also an

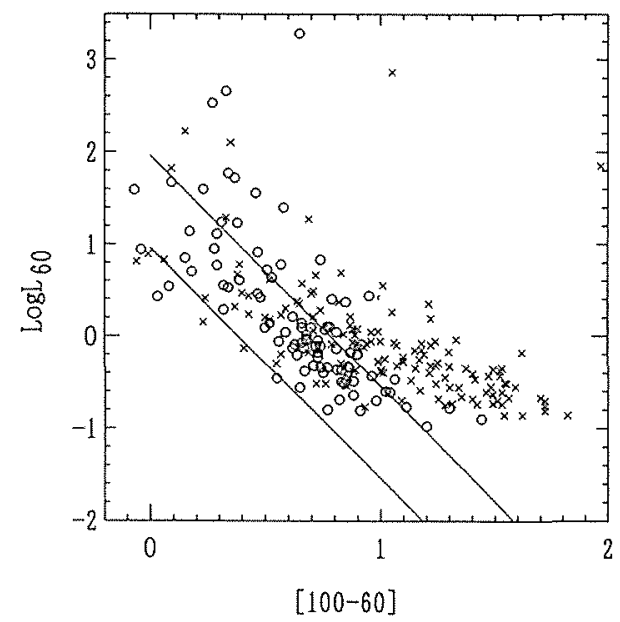

Fig. 4. FIR H-R diagram of all of the selected YSOs in Orion A and B. The circles are the YSOs with reliable fux densities at both 60 and $100 \mu \mathrm{m}$, while the crosses are the YSOs with upper limit flux densities at $100 \mu \mathrm{m}$.

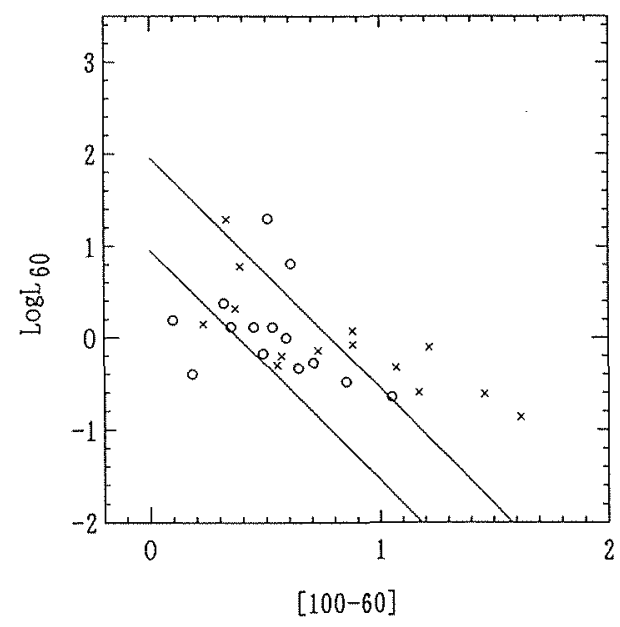

Fig. 5. FIR H-R diagram of 14 YSOs in Orion A. The crosses are plotted by using the data in IRAS PSC, the flux densities of which are upper limits at $100 \mu \mathrm{m}$. The circles are plotted by using the coadded data of the same objects obtained by Chen et al. (1993) who found the reliable flux densities at $100 \mu \mathrm{m}$. 
extended source in IRAS $12 \mu \mathrm{m}$ emission. Such IRAS point sources thus contain contribution from several or more YSOs. Those sources are indicated by bigger dashed-circles in the right panels of Figure 3 and are excluded from further analysis of the FIR H-R diagram.

\subsection{Fundamental Properties of the FIR $H-R$ Diagrams}

Dust mass in the FIR emitting envelope is evaluated by assuming that the envelope is optically thin in FIR and the dust is heated by the central source (Hildebrand 1983). Following Hildebrand and WAL, we assume that in envelope the density of dust grains is $3 \mathrm{~g} \mathrm{~cm}^{-3}$, the grain size $0.1 \mu \mathrm{m}$, the dust emissivity $7.5 \times 10^{-4}\left(\frac{125 \mu \mathrm{m}}{\lambda}\right)^{\beta}$, and the dust grains emit blackbody-like radiation with a temperature $T_{d}$ at FIR to millimeter wavelengths. Then the dust mass is estimated from the flux density at $60 \mu \mathrm{m}$ and the dust temperature as

$$
M_{d}=1.4 \times 10^{-6}(0.48)^{\beta} d^{2} f_{60}\left(e^{239.8 / T_{d}}-1\right) M_{\odot},
$$

where the distance $d$ is in $\mathrm{kpc}$ and the flux density $f_{60}$ in Jy. $\quad T_{d}$ is a function of the color [100-60] and $\beta$ (see equation (2)). Mass of the FIR emitting envelope, $M_{\text {env }}$ is given in Column 10 of Table 2 for each YSO, assuming a gas-to-dust ratio 100 and $\beta=1$. The solid lines in Figure 3 indicate the constant envelope mass. The slope of the line is independent on the values of $\beta$ and gas-to-dust ratio, and the envelope mass for $\beta=2$ is 1.7 times that for $\beta=1$. Comparing those with WAL's envelope masses, which were derived from the fitting of the spectra from 56 to $3,350 \mu \mathrm{m}$ of the eleven YSO's, we find a mean mass ratio, $M_{\text {ent }} / M_{W A L}=\operatorname{dex}(-0.06 \pm 0.23)$.

The spectrum of $M_{e n v}$ is shown in Figure 6 for each star-forming region, where the fraction of the extreme Class I YSOs (i.e., the YSOs with $L_{f} / L_{m}>4$ ), is illustrated in each bin. The spectrum of $L_{F I R}$ is also shown in Figure 7, where the fraction of the extreme Class I YSOs with active features are indicated in each bin.

The FIR H-R diagrams of Figure 3 are very similar to each other, although the FIR luminosities considerably differ among the star-forming regions, as shown in Figure 7. We recognize the following common properties of the FIR H-R diagrams:

(a) The colors [100-60] of the YSOs range from 0 to 1.2. Especially, the brightest YSOs in each star-forming region are in the range of $[100-60]=0$ to 0.5 .

(b) The active YSOs are most luminous in each star-forming region and its colors [10060 ] are less than 0.5 , i.e., $45 \mathrm{~K}>T_{d}>30 \mathrm{~K}$ for $\beta=1$.

(c) In each star forming region, the more luminous YSOs the higher dust temperatures, $T_{d}$, as deduced in $\S 1$, nearly along the constant "envelope mass" lines. We consider that these lines represent a fundamental sequence on the FIR $\mathrm{H}-\mathrm{R}$ diagram. We can it constant envelope mass sequence (CEMS).

(d) The extreme Class I YSOs (filled squares in the left panels of Figure 3) tend to belong to the higher CEMS. The property is also illustrated in Figure 6, where it is clear that the higher $M_{e n v}$ has the higher fraction of the extreme Class I YSOs.

(e) The extreme Class I YSOs are mostly invisible. On the other hand, the YSOs with $L_{f} / L_{m}<4$ tend to be associated with visible star as compared with the YSOs with $L_{f} / L_{m}>4$. The trend is shown in Table 3 . 


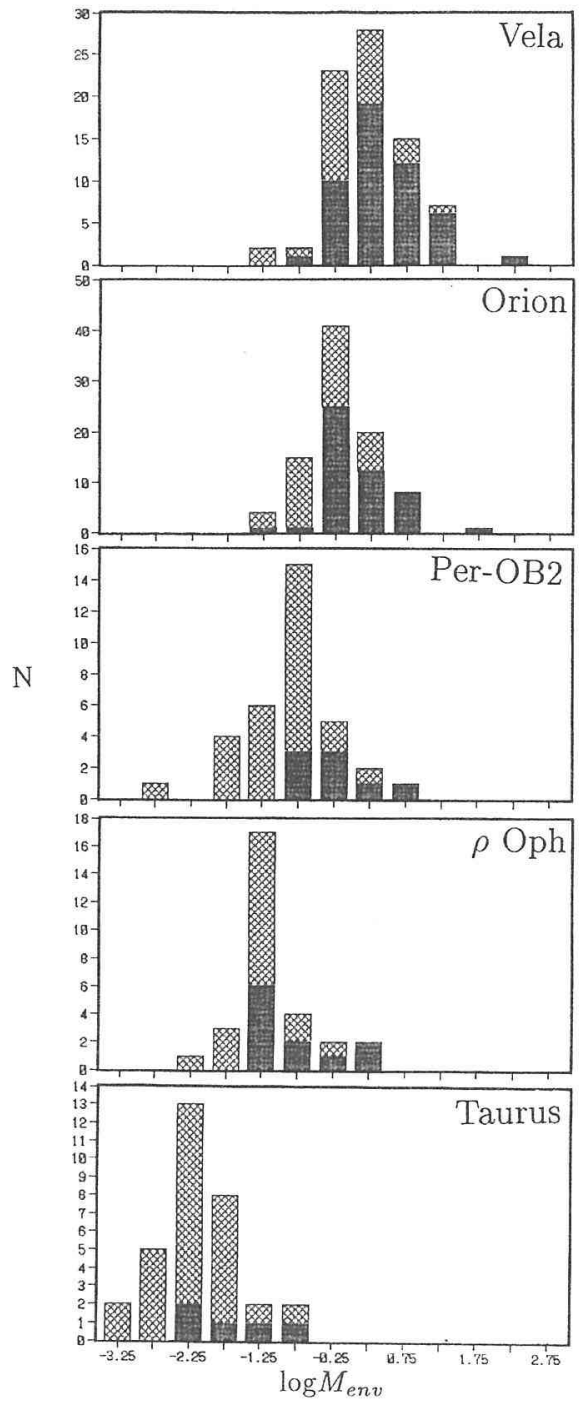

Fig. 6. Distribution of mass of the FIR emitting envelopes for the YSOs plotted in the FIR H-R diagrams (Figure 3). Heavy hatch indicates the YSOs with $L_{f} / L_{m}>4$, i.e., the extreme Class I.

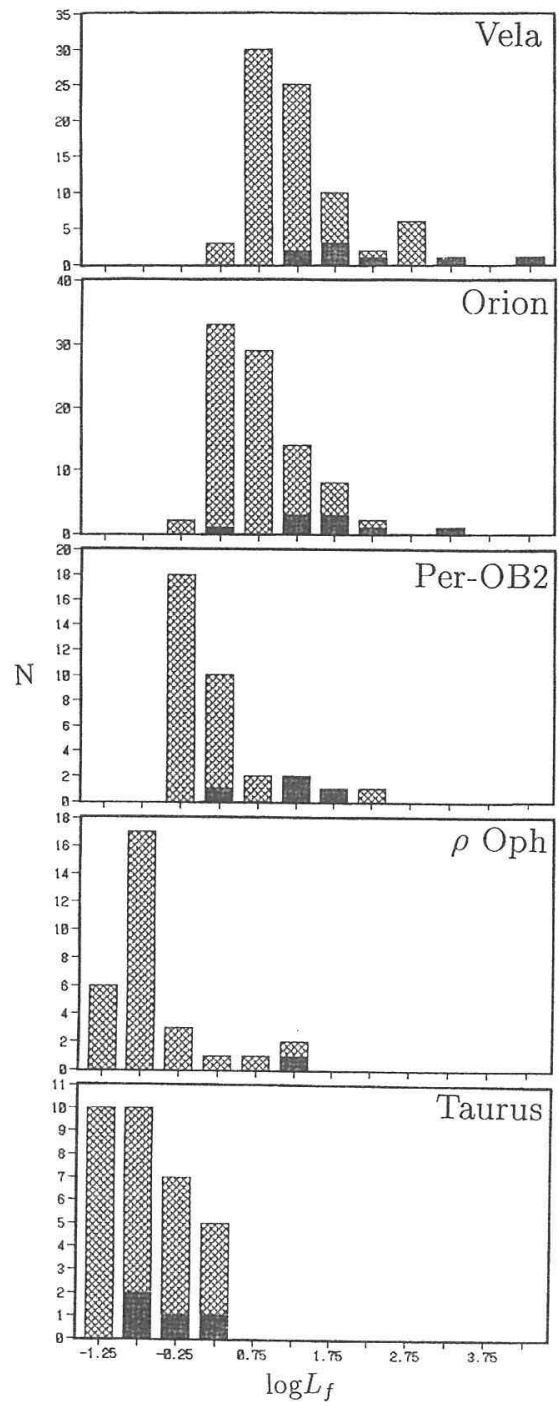

Fig. 7. Distribution of the FIR luminosities for the YSOs plotted in the FIR H-R diagrams (Figure 3). Heavy hatch indicates the extreme Class I YSOs with active features.

The property (a) has been well-known (Beichman et al. 1986; Emerson 1987; Wouterloot, Walmsley \& Henkel 1988). The property (b) has been also recognized. The active YSOs are most luminous in the individual star-forming regions (e.g., Fukui 1988; Myers et al. 1988; Snell at al. 1988; Cabrit \& André 1991; Chen et al. 1993). The IRAS point sources with CO outflows are listed in Berrili et al. (1989) and Fukui (1989) ; the luminosities mostly range in $1-10^{5} L_{\odot}$ and the average value and standard deviation of the colors [100-60] are $0.18 \pm 0.22$ for 31 sources with reliable IRAS flux densities in the former sample and $0.27 \pm 0.19$ for 102 sources in the latter sample. 
Wood \& Churchwell (1989) find the IRAS color [100-60] of 30 ultracompact H II regions to be $0.26 \pm 0.14$. Wouterloot \& Walmsley (1986) show that $I R A S$ sources with $\mathrm{H}_{2} \mathrm{O}$ masers mostly have $[100-60]=0$ to 0.6 . The properties (c) $-(\mathrm{e})$ have been partly recognized in relation with YSOs evolution, as shall be shown in $\$ 3.2$.

Besides the similarities mentioned above, we also notice the following differences among the FIR H-R diagrams of the individual clouds, which are mainly related to the YSOs masses and their evolutionary stages:

(i) The FIR luminosities $L_{f}$ and the masses $M_{e n v}$ of the FIR emitting envelopes: The difference of these values among the star-forming regions are clearly seen in Figures 6 and 7 ; the peaks of both the $\log M_{\text {env }}$ and $\log L_{f}$ spectra become less in order of the Vela, Orion, Per-OB2, $\rho$-Ophiucus, and Taurus. The peak values are listed in Table 4 and plotted by open squares in Figure 8 ; both the values, except for Taurus, are proportional to each other in an order of 1.5 , i.e., $\left(M_{e n v}, L_{f}\right)=\left(1.8 M_{\odot}, 5.6 L_{\odot}\right)$ in Vela to $\left(0.056 M_{\odot}, 0.18 L_{\odot}\right)$ in $\rho$-Ophiucus. For Taurus, the deviation from the correlation may be due to the higher fraction of evolved YSOs, i.e., the lower fraction of the extreme Class I YSOs (see Figure 6). We consider that the median of $M_{e n v}$ for the extreme Class I YSOs in each star-forming region represents a typical $M_{e n v}$ of the highest-mass YSOs in that region, and the median of $L_{f}$ for the active extreme Class I YSOs corresponds to a typical luminosity in the most luminous phase of such YSOs. These values are also listed in Table 4 and plotted in Figure 8 (filled squares), except for $\rho$ Ophiucus. These $\log M_{e n v}$ and $\log L_{f}$ decrease nearly linearly from Vela to Taurus, i.e., $\left(M_{e n v}, L_{f}\right)=\left(2.5 M_{\odot}, 158 L_{\odot}\right)$ in Vela to $\left(0.01 M_{\odot}, 0.3 L_{\odot}\right)$ in Taurus.

(ii) The fraction of extreme Class I YSOs : In Taurus the fraction is clearly less

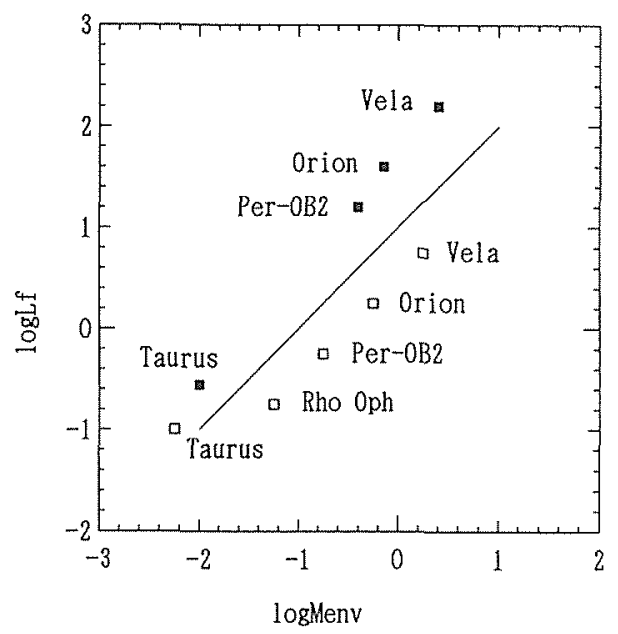

Fig. 8. Open squares : a correlation between the peaks of distributions of the envelope mass and FIR luminosity of YSOs. The mass and luminosity are in solar units. Filled squares : a correlation between the median of envelope masses of the extreme Class I YSOs and the median of the FIR luminosities of the active extreme Class I YSOs. The line indicates $L_{f}=10 M_{e n v}$ in solar units. 
Table 4. Characteristic FIR Luminosity and Envelope Mass of YSOs in the Five Star-Forming Regions ${ }^{1)}$

\begin{tabular}{llllclc}
\hline \hline & Per-OB2 & Taurus & Orion & Vela & $\rho$-Oph \\
\hline $\log L_{f}$ at peak & -0.25 & -1.0 & 0.25 & 0.75 & -0.75 \\
$\log M_{\text {en }}$ at peak & -0.75 & -2.25 & -0.25 & 0.25 & -1.25 \\
$\gamma$ index of mass spectrum & -1.8 & -1.6 & -1.7 & -1.6 & -1.6 \\
Fraction of extreme Class I & 0.24 & 0.16 & 0.54 & 0.63 & 0.38 \\
Median of $\log L_{f}^{2)}$ & $1.2(4)$ & $-0.56(4)$ & $1.6(9)$ & $2.2(8)$ & $1.2(1)$ \\
Median of $\log M_{\text {enr }}{ }^{3)}$ & $-0.4(8)$ & $-2.0(5)$ & $-0.15(48)$ & $0.4(49)$ & $-1.1(11)$ \\
$\gamma$ index of mass spectrum & & - & - & -1.5 & -1.5 & - \\
\hline
\end{tabular}

1) See Figures 6 and 7. The luminosity and mass are in solar units. 2) For the extreme Class I YSOs with active features. The numbers in paranthesis are the YSOs' number. 3) For the extreme Class I YSOs. The numbers in parathesis are the YSOs' number.

than in the other star forming regions (see Figure 6 and Table 4). This difference must be related to the evolutionary stage of star formation in the individual clouds. But the statistics in each star-forming region are imcomplete due to existence of many YSOs with $\mathrm{FQ}_{100}=1$ (the second group of Table 2) and probably many colder YSOs with $\mathrm{FQ}_{60}=1$ and $\mathrm{FQ}_{100} \geq 2$ which are not taken into account in this study.

\section{Discussion}

\subsection{Mass of the FIR Emitting Envelope}

The mass spectrum of molecular cores in a star-forming region has an index between -1.7 and -1.4 , which are different from the index of stellar mass of -2.35 (Blitz 1991; Tatematsu et al. 1993). The mass spectra of the FIR emitting envelope shown in Figure 6 have indexes of $\gamma=-1.8$ to -1.6 (see Table 4). The indexes are also evaluated for the mass spectra of the extreme Class I YSOs in Orion and Vela and their values are both $\gamma=-1.5$. Those are similar to the indexes of molecular cores.

Chen et al. (1993) measured column densities of ${ }^{13} \mathrm{CO}(J=1-0)$ with a beam size of $2.7^{\prime}$ on IRAS point sources in Orion A. They also obtained the IRAS flux densities by making co-added images. By using their flux densities at $60 \mu \mathrm{m}$ and $100 \mu \mathrm{m}$ with the uncertainties less than $50 \%$, we found values of [100-60], $L_{60}, L_{f} / L_{m}$, and $M_{e n v}$ of the point sources. We illustrate in Figure 9 the correlation between the $M_{\text {eni }}$ and $N\left({ }^{13} \mathrm{CO}\right)$ for the sources with $N\left({ }^{13} \mathrm{CO}\right)>0.4 \times 10^{16} \mathrm{~cm}^{-2}$. Figure 9 shows that $M_{e m v}$ of the YSOs with $L_{f} / L_{m}>4$ increase with $N\left({ }^{13} \mathrm{CO}\right)$, while the YSOs with $L_{f} / L_{m}<3$ deviate from this correlation which must be due to decreasing of dust mass and molecular mass with evolution. The correlation seems to be $N\left({ }^{13} \mathrm{CO}\right) \propto M_{e n v}^{0.6}$. The molecualr core masses of three objects in Figure 9 were derived by Chen, Fukui \& Yang (1992) and the masses are several times larger than $M_{\text {env }}$.

We show in Figure 10 a correlation between $M_{e n v}$ and $L_{f}$ for the YSOs plotted in all of the FIR H-R diagrams of Figure 3. We find in this diagram that the more luminous YSOs which are making higher mass stars tend to have the higher envelope mass, but the sequence of the YSOs with $L_{f} / L_{m}>4$ (filled squares) separates from that of the 


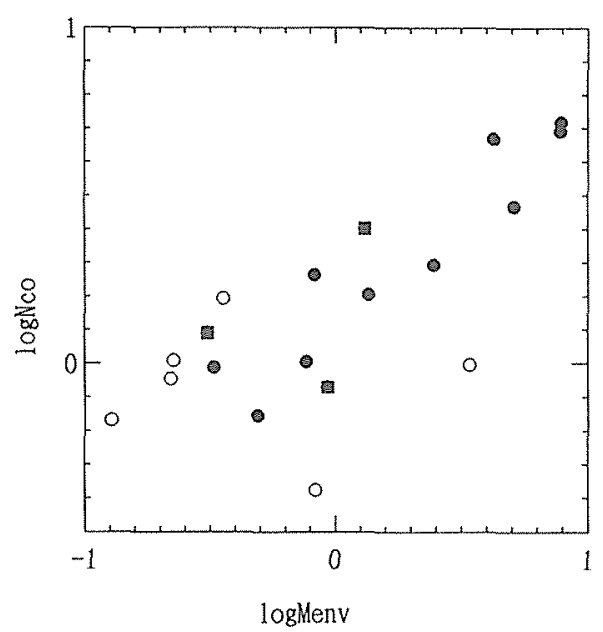

Fig. 9. A correlation between mass, $M_{\epsilon n}\left(M_{\odot}\right)$, of the FIR emitting envelope and ${ }^{13} \mathrm{CO}$ $U=1-0)$ column density in unit of $10^{16}$ $\mathrm{cm}^{-2}, N\left({ }^{13} \mathrm{CO}\right)$, of YSOs in Orion $\mathrm{A}$. The $N\left({ }^{13} \mathrm{CO}\right)$ were obtained by Ghen et al. (1993) in a $2.7^{\prime}$ beam size. The filled circles indicate YSOs with $L_{f} / L_{m}>4$, filled squares YSOs with $L_{f} / L_{m}>3$, and the open circles YSOs with $L_{f} / L_{m}<3$.

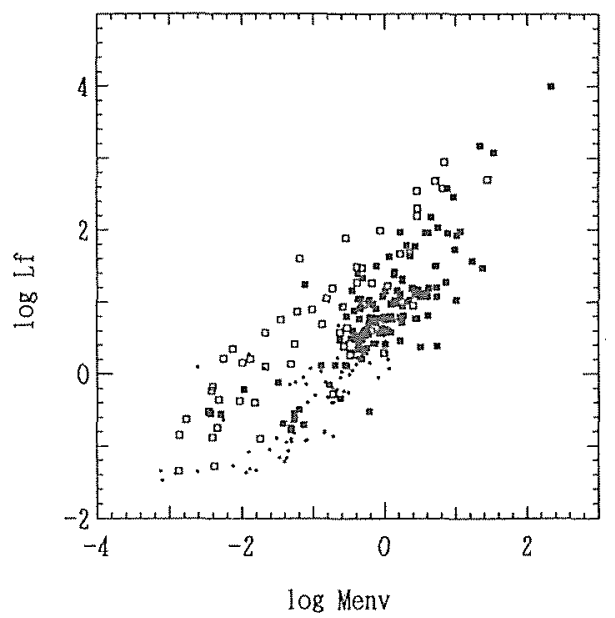

Fig. 10. A correlation between the envelope mass and the FIR luminosity for the individual YSOs plotted in the FIR $\mathrm{H}-\mathrm{R}$ diagrams (Figure 3 ). The filled squares are $Y S O s$ with $L f / L_{m}>4$, the open squares YSOs with $L_{f} / L_{m}<4$, and the dots YSOs with unknown $L_{f} / L_{m}$ value.

YSOs with $L_{f} f L_{m}<4$ (open squares). The YSOs move from the lower sequence to the upper one as they are evolving. As shown in the mass spectrum of each star forming region in Figure 6, the YSOs decrease the envelope mass by a half to one order of magnitude with evolution from the phase of $L_{f} / L_{m}>4$ to $<4$. If the decreasing of $L_{f}$ also occurs with a rate similar to that of $M_{\text {env }}$, the separation of sequences in Figure 10 is not realized. If $M_{e m}$ decreases without decreasing of $L_{f}$, the separation between the two sequences in Figure 10 means that the $M_{e n v}$ decrement is about one order of magnitude for YSOs with $L_{f} \sim 1 L_{\odot}$ to $10 L_{\odot}$, and the rate seems to be less for the YSOs with higher $L_{f}$. This is consistent with the results deduced from the mass spectrum of each star-forming region. André \& Montmerle (1994) found a similar trend from their $1.3 \mathrm{~mm}$ observations of YSOs in $\rho$-Ophiucus.

Assuming that the resulting stellar mass is $-0.5 M_{\odot}$ in Taurus and $\sim 4 M_{\odot}$ in Orion and the typical envelope masses are the medians of the extreme YSOs shown in Table 4, we obtain $M_{\text {env }} / M_{\text {star }} \sim 0.02$ and $\sim 0.2$ for YSOs in Taurus and Orion, respectively. Since $M_{\text {end }} / M_{\text {star }}<1$ even at the extreme Class I stage, these YSOs seem to have already stored materials of stellar mass at the central parts in this evolutionary stage (André \& Montmerle 1994).

\subsection{Evolutionary Sequence}

The FIR H-R diagram describes the evolutionary sequence of YSOs which are usually accepted based on many observational and theoretical works. The birth of 
protostars begins by infalling of inner part of molecular cloud cores. The main feature at this first stage is the increasing of luminosity and the growing of central objects (star+disk). The radiation is emitted mainly at FIR wavelength. This evolutionary sequence of YSOs is represented by the CEMS on the FIR H-R diagram, i.e., the common property (c); the YSOs move upward along the CEMS with increasing the 60 $\mu \mathrm{m}$ luminosity, raising the temperature of the envelope, and keeping the mass of the FIR emitting envelope nearly constant. The increase of mass and luminosity of the central objects induce at last the activities such as molecular outflow, $\mathrm{H}_{2} \mathrm{O}$ maser, and compact H II region (the common property (b) of the FIR H-R diagrams). During and after the active phase, the (dust) mass of the FIR emitting envelope reduces and the SEDs of YSOs extend toward mid- and near-infrared wavelengths as well as decreasing the FIR luminosities, and the central stars become visible. The trends appear in the FIR H-R diagrams as the common properties (d) and (e).

To delineate specifically the evolutionary sequence in the early, pre-active stage and also post-active stage, we examine the FIR H-R diagram of each star-forming region (Figure 3) using other observational data of YSOs.

\section{Per-OB2}

In the Per-OB2 both low- and high-mass stars have formed (Bachiller, MartinPintado \& Planesas 1991; Ladd et al. 1993). This appears also in the FIR H-R diagram in Figure 3. The five luminous sources with $\log L_{60}>0.4$ are the high-mass YSOs with $M_{\text {env }} \sim 1 M_{\odot}$, while the others are low-mass YSOs. The three luminous YSOs, SVS 3 at $\left([100-60], \log L_{60}\right)=(0.24,1.82)$, RNO 15 FIR at $(0.29,0.77)$ and L1448 IRS3 at $(0.85,0.45)$, are associated with H II regions (references are shown in Table 2). RNO 15 FIR shows a CO outflow with "age" $2.2 \times 10^{4}$ yr (Fukui 1989 and references therein). Roger \& Dewdney (1992) suggest that SVS3 is B3.5 ZAMS. L1448 IRS3 is B3 ZAMS with outflow of "age" $-3.5 \times 10^{3} \mathrm{yr}$ (Anglada et al. 1989; Bachiller et al. 1990). Their $M_{e n v} / M_{\text {star }}$ are thus $\sim 0.2-0.3$.

Bachiller et al. (1991) found outflows in IRAS $03271+3013$ and $03282+3035$ associated with T Tauri stars (TTSs) $\left(-2-3 M_{\odot}\right)$; the latter is located at $(0.79,-0.57)$, and the outflow is young $\left(\sim 1.6 \times 10^{4} \mathrm{yr}\right)$ and unusual jet-like $(\eta \sim 70 \mathrm{~km} / \mathrm{s})$. About ten YSOs with $M_{e n} \sim 0.1 M_{\odot}$ and $\log L_{60}<-0.5$ are mostly invisible and probably the extreme Class I YSOs; they may be young, except for a TTS LkH- $\alpha 325$ at $(0.93$, -1.09). The YSOs with $M_{e n v}<0.1 M_{\odot}$ and $T_{d}>30 \mathrm{~K}$ for $\beta=1$, on the other hand, tend to have $L_{f} / L_{m}<4$, and they are older. One of these is a TTS RNO 13 at $(0.38$, -0.30 ) showing an outflow with "age" $2.3 \times 10^{4} \mathrm{yr}$ (Fukui 1989 and references therein).

\section{Tauras}

In the Taurus star-forming region, the YSOs luminosities at FIR are mostly lower than those in Per-OB2 (see Figure 7). T Tauri stars have masses less than $1 M_{\odot}$ in Taurus (Beckwith et al. 1990) and $-2-3 M_{\odot}$ in Per-OB2 (Bachiller et al. 1991). The FIR H-R diagram indicates that most of the YSOs in Taurus have $L_{f} / L_{m}<4$ and the fraction of visible stars is higher than in Per-OB2. Furthermore, the selected YSOs with $\mathrm{FQ}_{100}=1$ in Taurus (the second group in Table 2) are mostly the TTSs with the Class II SEDs. Thus, the YSOs in Taurus are in the later evolutionary stages than 
those in Per-OB2.

The embedded YSO (IRAS $04368+2557)$ assciated with L1527 is at $(0.61,-0.46)$ on the FIR H-R diagram and presumably the youngest active YSO in our sample of the Taurus. The value of $L_{f} / L_{m}>19.5$ is very large and its SED shows a single peak modified blackbody for $T=30 \mathrm{~K}$ with $L_{F I R}=1.8 L_{\odot}$ (Ladd et al. 1991). L1527 is a dense $\mathrm{NH}_{3}$ core with a radius of $0.08 \mathrm{pc}$ and a core mass of $2.4 M_{\odot}$ (Benson \& Myers 1989). The core mass is -20 times larger than the $M_{e n v}$, implyng that the molecular core radius is one order of magnitude larger than that of the FIR emitting region.

Ohashi et al. (1991) detected CS $(J=2-1)$ emission associated with L1527 and also the bright YSOs with the higher dust temperatures, IRAS $04169+2702$ at $(0.02$, -0.49), IRAS $04240+2559$ (DG Tau) at $(0.08,-0.12)$, and IRAS $04361+2547$ (TMR-1) at $(-0.08,-0.09)$, but not detected for the less bright TTSs, IRAS $04189+2650$ (FS Tau) and $04306+2514$ (DL Tau) with $\log L_{60}=-0.86$ and -1.55 , respectively.

Table 5. T Tauri Stars with Similar Mass in Taurus

\begin{tabular}{lccccccc}
\hline \hline \multicolumn{1}{c}{ TTs } & IRAS Name & {$[100-60]$} & $\log L_{60}$ & $\log L_{f}$ & $\log M_{e n v}$ & $M_{*}\left(M_{\odot}\right)^{1)}$ & $\log (\mathrm{age})^{1)}$ \\
\hline DG Tau & $04240+2559$ & 0.08 & -0.12 & 0.20 & -1.88 & 0.56 & 5.47 \\
Haro 6-13 & $04292+2422$ & 0.14 & -0.85 & -0.52 & -2.46 & 0.55 & 5.10 \\
HK Tau & $04288+2417$ & 0.34 & -1.29 & -0.88 & -2.40 & 0.55 & 5.94 \\
DP Tau & $04395+2509$ & 0.55 & -1.80 & -1.28 & -2.38 & 0.60 & 6.20 \\
\hline
\end{tabular}

1) Star's mass in solar unit and age in year of Beckwith et al. (1990).

The mean ages of TTSs of Beckwith et al. (1990) are $9.2 \times 10^{5} \mathrm{yr}$ for three TTSs with color $[100-60]>0.3$ and $7.9 \times 10^{5} \mathrm{yr}$ for five TTSs with color $[100-60]<0.3$. Especially, when we compare four TTSs with similar stellar mass in Table 5, the positions of three TTSs on the FIR H-R diagram move with age toward larger [100-60] and lower $\log L_{60}$. These facts suggest an evolutionary path of the TTSs stage on the FIR H-R diagram that both the $60 \mu \mathrm{m}$ luminosity and the dust temperature of the envelope decrease along the lower CEMS.

There is other observational evidence supporting this trend on the evolutionary path of pre-main sequence stars. Clark (1991) examined 205 nearby IRAS point sources, which are thought to be pre-main sequence stars, and found that in accordance with increasing of the separation from the molecular core, presumably with age (Myers et al. 1987), the colors [100-60] increase from 0.2 to 0.85 and the infrared luminosities decrease about one order of magnitude. Beckwith et al. (1990) find from the $1.3 \mathrm{~mm}$ continuum emission of TTSs that the circumstellar dust masses do not decrease with age up to $10^{7} \mathrm{yr}$. They also show that the $60 \mu \mathrm{m}$ flux densities decrease with decreasing dust temperature and the older TTSs tend to have lower dust temperatures. Beckwith et al. (1990) and Clark (1991) suggest the reasons of these trends as due to decreasing of their luminosity and their energetic activity, such as accretion, with time. 


\section{Orion}

There are two brightest YSOs with $\log L_{60}>2$; one is IRAS 05391-0217 (NGC 2023) which is an infrared cluster and an extended source in $100 \mu \mathrm{m}$ emission, and another is IRAS 05327-0457 which is probably the most massive YSO in our sample. Excluding the two objects, the YSOs in the FIR H-R diagram seem to be divided into two groups by $M_{\text {env. }}$. Wiramihardja et al. (1991) show that both high- and low-mass YSOs co-exist in the Orion cloud.

The YSOs along the CEMS of $M_{\text {env }} \sim 5 M_{\odot}$ are the extreme Class I and thus are in early evolutionary stage. The brightest of these is NGC $2068 \mathrm{H}_{2} \mathrm{O}$ at $(0.34,1.77)$ which has an outflow with the "age" of $2 \times 10^{5} \mathrm{yr}$ (Edwards \& Snell 1984). IRAS $05335-0645$ at $(0.85,0.37)$ is associated with three TTSs (Weintraub 1990), but it has a high $L_{f} / L_{m}(>8.1)$. Around the CEMS of $M_{e n v} \sim 1 M_{\odot}$, there are five visible YSOs with $L_{f} / L_{m} \leq 4$ (open squares on the left panel). One of these YSOs is a Herbig Ae/Be star N'SK 81 at $(0.78,0.10)$. Those YSOs are probably high-mass YSOs evolved from the extreme Class I YSOs with $M_{e n v} \sim 5 M_{\odot}$.

There are many invisible extreme Class I YSOs around the CEMS of $M_{e n v} \sim 1 M_{\odot}$, while the YSOs with $L_{f} / L_{m}<4$ are distributed nearly along the CEMS of $M_{e n v} \sim 0.2 M_{\odot}$. One of the visible YSOs, IRAS $05464+0106$ at $(0.64,-0.21)$, is a TTS (GregorioHetem et al. 1992), and three other visible YSOs around the CEMS of $M_{\text {env }} \sim 0.2 M_{\odot}$ are likely to be TTSs (Wiramihardja et al. 1991, 1993). These TTSs are probably YSOs evolved from the extreme Class I YSOs with $M_{e n v} \sim 1 M_{\odot}$.

\section{Vela}

On the left panel in Figure 3, the extreme Class I YSOs (filled squares) are distributed along the GEMS of $\sim 10 M_{\odot}$ and $\sim 1 M_{\odot}$. These are high-mass YSOs in the early evolutionary stage. The most massive group contains a Herbig Ae/Be star RCW 34 at $(0.19,2.82)$ which is associated with $\mathrm{H}$ II region, bright nebula and $\mathrm{H}_{2} \mathrm{O}$ maser. The brightest YSOs inVela, IRAS 08576-4334 at $(0.27,3.62)$ is associated with a Herbig Ae/Be star, $\mathrm{H}$ II region, bright nebula, $\mathrm{H}_{2} \mathrm{O}$ maser and probable outflow RCW 36 ; this is an extended source at the IRAS four bands and probably a complex of massive YSOs. Liseau et al. (1992) claim that the most massive stars in the Vela starforming region are the early B-type. Thus the $M_{\text {env }} / M_{\text {star }}$ is close to unity for such massive stars.

$\rho-O p h$

The YSOs in this star-forming region are mostly less luminous and have cold envelopes. The envelope masses are larger than YSOs in Taurus (see also Figure 6) but the FIR luminosites are similar to YSOs in Taurus (see Figure 7). Kenyon et al. (1990) show that the luminosity function for Class I and Glass II sources in $\rho-O p h$ is similar to that in Taurus-Aurigae, but the fraction of Class $I$ sources is larger in $\rho$-Oph than in Tau-Aur. These also suggest that $M_{\text {env }}$ of low-mass YSOs reduces by one order of magnitude during the evolution from the extreme Class I stage to TTS stage.

The two brightest YSOs with $\log L_{60}>0.4$ seem to be more massive than others. One of these IRAS $16293-2422\left(\rho-O\right.$ ph-S) at $(0.61,0.60)$ has $L_{f} / L_{m}>158$ which is the 
largest value in our sample of YSOs listed in Table 2. For this YSO, André et al. (1990) obtained $M_{\text {ent }} \sim 0.41 M_{\odot}$ from the observation at $1.3 \mathrm{~mm}$, and Wilking et al. (1989) give the infrared emitting mass to be $2 M_{\odot}$. Lada (1991, and reference therein) showed that its SED fits a single temperature modified blackbody ( $T=35 \mathrm{~K}$ for $\beta=1.5$ ), and suggested that the YSO is associated with edge-on bipolar outflows.

In the four FIR H-R diagrams, except for that of Taurus, the extreme Class I YSOs are more at $[100-60]>0.5$ than at $[100-60]<0.5$. The trend implies that the life time in the cold, less luminous extreme Class I YSOs stage is comparable to or rather longer than that in the luminous active phase. This result differs from that of the spherical symmetric infalling model (e.g., Yorke 1979; Stahler et al. 1980), and supports the model that the gas accretion onto the central star slowly occurs through the circumstellar disk (Terebey at al. 1984; Shu et al. 1987).

\section{Summary}

The FIR H-R diagrams of YSOs are constructed for each of six nearby starforming regions by using the IRAS flux densities at 60 and $100 \mu \mathrm{m}$. The FIR H-R diagrams are characterized by a constant envelope mass sequence (CEMS) along which YSOs change the dust temperatures in the envelope in accordance with the luminosities of the central objects keeping the envelope mass and radius nearly constant.

The evolution of the YSOs is traced along the GEMS ; (1) in the early phase YSOs, i.e., the extreme Class I YSOs, move upward along the CEMS with increasing the luminosity of the central object and the dust temperature of the envelope, (2) the YSOs become active at the brightest phase and reduce the mass of envelope, and (3) after the active phase the FIR luminosity decreases with time and the dust temperature of the envelope becomes lower, i.e., the YSOs move downward along the lower CEMS.

Analyzing the FIR H-R diagrams of the nearby star-forming regions, we suggest the extreme Class I YSOs have already stored most of the material of stellar mass at the central part and are forming stars by a slow accretion of the material.

We may use the FIR H-R diagrams to specify the evolutionary state of a YSO and the resulting stellar mass. The FIR H-R diagrams must be made in future by FIR observations with higher sensitivity and spatial resolution for many other star-forming regions.

One of us (TD) thanks the Japanese Ministry of Education, Science, and Culture for the scholarship.

\section{References}

Adams, F.C., ApJ, 363, 578, 1990.

Adams, F.C. and Shu, F.H., ApJ, 296, 655, 1985.

Adams, F.C., Lada, C.J. and Shu, F.H., ApJ, 312, 788, 1987.

André, P. and Montmerle, T., ApJ, 420, 837, 1994.

André, P., Montmerle, T., Feigelson, E.D. and Steppe, H., A\& A, 240, 321, 1990.

Anglada, G., Rodriguez, L.F., Torrelles, J.M., Estalella, R., Mo, P.T.P., Canto, J., Lopez, R. and 
Verdes-Montenegro, H., ApJ, 341, 208, 1989.

Bachiller, R., Cernicharo, J., Martin-Pintado, J., Tafalla, M. and Lazareff, B., $A$ \& A, 231, 174, 1990.

Bachiller, R., Martin-Pintado, J. and Planesas, P., $A \& A, 251,639,1991$.

Beckwith, S., Sargent, A.I., Scoville, N.Z., Masson, G.R., Zuckerman, B. and Phillips, T.G., ApJ, 309, $755,1986$.

Beckwith, S.V.W., Sargent, A.I., Chini, R.S. and Guesten, R., AJ, 99, 924, 1990.

Beichman, C.A., Myers, P.C., Emerson, J.P., Harris, S. Mathieu, R., Benson, P.J. and Jenning, R.E., ApJ, 307, 337, 1986.

Benson, P.J. and Myers, P.C., ApJS, 71, 89, 1989.

Berrili, F., Ceccarelli, C., Liseau, R., Lorenzetti, D., Saraceno, P. and Spinoglio, L., MNRAS, 237, 1, 1989.

Blitz, L. in The Physits of Star Formation and Early Stellar Evolutirn, eds. C.J. Lada \& N.D. Kylafis (Dorbrecht: Kluwwer), p. 3, 1991.

Braz, M.A. and Scalise, Jr., E., A\& A, 107, 272, 1982.

Butner, H.M., Evans II, N.J., Harvey, P.M., Mundy, L.G., Natta, A. and Randich, M.S., ApJ, 364, $164,1990$.

Carbrit, S. and André, P., ApJ, 379, 125, 1991.

Carpenter, J.M., Snell, R.L., Schloerb, F.P. and Skrutskie, M.F., ApJ, 407, 657, 1993.

Cesaroi, R., Palagi, F., Feli, M., Catarzi, M., Comoretto, G., Di Franco, S., Giovanadri, C. and Palla, F., $A \& A S, 76,445,1988$.

Chen, H., Fukui, Y. and Yang, J., ApJ, 398, 544, 1992.

Chen, H., Tokunaga, A.T. and Fukui, Y., ApJ, 416, 235, 1993.

Chen, H., Tokunaga, A.T., Strom, K.M. and Hodapp, K.W., ApJ, 407, 639, 1993.

Chini, R., Kresa, E., Mezger, P.G. and Gemund, H.P., $A$ \& $A$, 154, L8, 1986.

Chini, R., Krügel, E. and Kreysa, E., $A \& A, \mathbf{1 6 7}, 315,1986$.

Churchwell, E., Wolfire, M. G. and Wood, D.O.S., ApJ, 354, 247, 1990

Clark, F.O., ApJS, 75, 611, 1991

Dame, T.M., Ungerechts, H., Cohen, R.S., de Geus, E.J., Grenier, I.A., Ma, J., Murphy, D.C., Nyman, L.-A. and Thaddeus, P., ApJ, 322, 706, 1987

Davidson, J.A., ApJ, 315, 602, 1987.

de Geus, E.J., Bronfman, L. and Thaddeus, P., $A \& A, 231,137,1990$.

Edwards, S. and Snell, R.L., ApJ, 281, 237, 1984.

Ellis, Jr., H.B., Lester, D.F., Harvey, P.M., Joy, M., Telesco, C.M, Decher, R. and Werner, M.W., ApJ, 365, 287, 1990.

Emerson, J.P., in Star Forming Regins, eds, M. Peimbert and J. Jugaku (Drdrecht: D. Reidel), p.19, 1987.

Emerson, J.P., in Formation and Evolution of Low Mass Starss, eds. A.K. Dupree and M.T.V.T. Lago (Dordrecht: Kluwer), p. 21, 1988a.

Emerson, J.P., in Formation and Evolution of Low Mass Starss, eds. A.K. Dupree and M.T.V.T. Lago (Dordrecht: Kluwer), p. 193, 1988b.

Fukui, Y., Vistas Astron., 31, 217, 1988.

Fukui, Y., in Low Mass Star Formation and Pre-Mains Sequence Objects, ed. Bo Reipurth, (ESO, Garching), p. 95, 1989.

Gregorio-Hetem, J., Lepine, J.R.D., Quast, G.R., Torres, C.A.O. and de la Rea, R., AJ, 103, 549, 1992.

Gürtler, J. Henning, Th., Krügel, $\mathrm{E}$ and Chini, R., $A \& A, 252,801,1991$.

Harris, S., Clegg, P. and Hughes, J., MNRAS, 235, 441, 1988.

Harvey, P.M., Thronson, Jr., H.A. and Gatley, I., ApJ, 231, 115, 1979.

Henning, T., Cesaroni, R., Walmsley, M. and Pfau, W., A\& AS, 93, 525, 1992.

Heyers, M.H., Ladd, E.F., Myers, P.C. and Campbell, B., AJ, 99, 1585, 1990.

Hildebrand, R.H. QJRAS, 24, 267, 1983.

IRAS Explanatory Supplement, eds. C.A. Beichman, G. Neugebauer, H.A.J. Habing, P.E. Clegg and T.J. Chester (Washington, DC: NASA), 1988.

IRAS Point Sources Catalog, Version 2.0, in Selected Astronomical Catalogs Vol. 1 (CD-ROM), 
Astronomical Data Center, NASA, 1986.

Kenyon, S.J., Galvet, N. and Hartmann, L., ApJ, 414, 676, 1993.

Kenyon, S.J., Hartmann, L.W., Strom, K.M. and Strom, S.E., AJ, 99, 869, 1990.

Lada, C.J., in Star Forming Regions, eds. M. Peimbert and J. Jugaku (Dordrecht: D. Reidel), p. 1, 1987.

Lada, C.J., in The Physics of Star Formation and Early Stellar Evolution, eds. C.J. Lada and N.D.

Kylafis (Dordrecht: Kluwer), p. 329, 1991.

Lada, E.A., Bally, J. and Stark, A.A., ApJ, 368, 432, 1991 a.

Lada, E.A., DePoy, D.L., Evans II, N.J. and Gatley, I., ApJ, 371, 171, 1991 b.

Ladd, E.F., Adams, G.C., Casey, S., Davidson, J.A., Fuller, G.A., Harper, D.A., Myers, P.C. and Padman, R., ApJ, 366, 203, 1991.

Ladd, E.F., Lada, E.A. and Myers, P.C., ApJ, 410, 168, 1993.

Larson, R.B., in Star Formation, ed. T. De Jong and Maeder, p. 249, 1977.

Leincrt, C. and Haas, M., ApJ, 342, L39, 1989.

Liseau, R., Lorenetti, D., Nisini, B., Spinoglio, L. and L. Monetti, A., A \& A, 265, 577, 1992.

Lorenzetti, D., Spinoglio, L. and Liseau, R., A\& A, 275, 489, 1993.

Maddalena, R.J., Morris, M., Moscowitz, J., and Thaddeus, P., ApJ, 303, 375, 1986.

Márque-Limon, A., Lopéz-Molina, M.G. and Chavarria-K, C., A\& AS, 95, 391, 1992.

McCutcheon, W.H., Dewdney, P.E., Purton, C.R. and Sato, T., AJ, 101, 1435, 1991.

Mezger, P.G., Wink, S.E., Zylka, R., $A \& A, 228,95,1990$.

Morgan, J.A., Schloerb, F.P. and Snell, R.L., ApJ, 376, 618, 1991.

Moriarty-Schieven, G.H., Wannier, P.G., Tamura, M. and Keene, J., ApJ, 400, 260, 1992.

Myers, P.C., Fuller, G.A., Mathieu, R.D., Beichman, G.A., Benson, P.J., Schild, R.E. and Emerson, J.P., $A p J, 319,340,1987$

Myers, P.C., Heyer, M., Sncll, R.L. and Goldsmith, P.F., ApJ, 324, 907, 1988.

Myers, P.C. and Ladd, E.F., ApJ, 413, L47, 1993.

Natta, A., Palla, R., Butner, H.M., Evans II, N.J. and Harvey, P.M., ApJ, 406, 674, 1993.

Ohashi, N., Kawabe, R., Hayashi, M. and Ishiguro, M., AJ, 102, 2054, 1991.

Palagi, F., Cesaroni, R., Comoretto, G., Felli, M. and Natale, V, A\& AS, 101, 153, 1993.

Roger, R.S. and Dewdney, P.E., ApJ, 385, 536, 1992.

Rowan-Robinson, M., ApJS, 44, 403, 1980.

Rucinski, S.M., AJ, 90, 2321, 1985.

Sargent, A.I., ApJ, 233, 164, 1979.

Scoville, N.Z. and Kwan, J., ApJ, 206, 718, 1976.

Shu, F.H., ApJ, 214, 488, 1977.

Shu, F.H., Adams, F.C. and Lizano, S., ARA \& A, 25, $23,1987$.

Snell, R.L., Huang, Y.L., Robert, L., Dickman, L. and Claussen, M.J., ApJ, 325, 853, 1988.

Stahler, S.W., Shu, F.H. and Taam, R.E., ApJ, 241, 637, 1980.

Strom, K.M., Margulis, M. and Strom, S.E., ApJ, 346, L33, 1989.

Strom, K.M., Strom, S.E. and Merrill, K.M., ApJ, 412, 233, 1993.

Tamura, M., Gatley, I., Waller, W. and Wernen, M.W., ApJ, 374, L25, 1991.

Tatematsu, K., Umemoto, T., Kaneya, O., Hirano, N., Hasegawa, T., Hayashi, M., Iwata, T., Kaifu, N., Mikami, H., Murata, Y., Nakano, M., Nakano, T., Ohashi, N., Sunada, K., Takaba, H. and Yamamoto, S., ApJ, 404, 643, 1993.

Terebey, S., Shu, F.H. and Cassen, P., ApJ, 286, 529, 1984.

Ungerechts, H. and Thaddeus, P., ApJS, 63, 645, 1987.

Walker, C.K., Adams, F.C. and Lada, C.J., ApJ, 349, 515(WAL), 1990.

Weintraub, D.A., ApJS, 74, 575, 1990.

Wilking, B.A., Lada, C.J. and Young, E.T., ApJ, 340, 823, 1989.

Wilking, B.A., Mundy, L.G., Blackwell, J.H. and Howe, J.E., ApJ, 345, 257, 1989.

Wiramihardja, S.D., Kogure, T., Yoshida, S., Ogura, K. and Nakno, M., PASJ, 41, 155, 1989.

Wiramihardja, S.D., Kogure, T., Yoshida, S., Nakano, M., Ogura, K. and Iwata, T., PASJ, 43, 27, 1991.

Wiramihardja, S.D., Kogure, T., Yoshida, S., Ogura, K. and Nakno, M., PASJ, 45, 643, 1993. 
Wood, D.O.S. and Churchwell, E., ApJ, 340, 265, 1989.

Wouterloot, J.G.A. and Brand, J., $A \& A S, \mathbf{8 0}, 149,1989$.

Wouterloot, J.G.A., Brand, J. and Fiegl, K., $A \& A S$, 98, 589, 1993.

Wouterloot, J.G.A., Henkel, C. and Walmsley, C.M., $A \& A, 215,131,1989$.

Wouterloot, J.G.A. and Walmsley, C.M., $A \& A, 168,237,1986$.

Wouterloot, J.G.A., Walmsley, C.M. and Henkel, C., $A \& A$, 203, 367, 1988.

Wynn-Wiliams, G.G., ARA \& A, 20, 587, 1982.

Yamada, T., Takata, T., Djamaluddin, T., Tomita, A., Aoki, K., Takeda, A. and Saitō, M., ApJS, 89, 1993.

Yorke, H.W., $A \& A, 80,308,1979$.

Yorke, H.W., $A \& A, 85,215,1980$.

Yorke, H.W. and Shustov, B.M., $A \& A, 98,125,1981$.

Zinnecker, H., in ESO Workshop on Low Mass Star Formation and Pre-mainsequence Objects (ed. B. Reipurth), p. 447, 1989. 